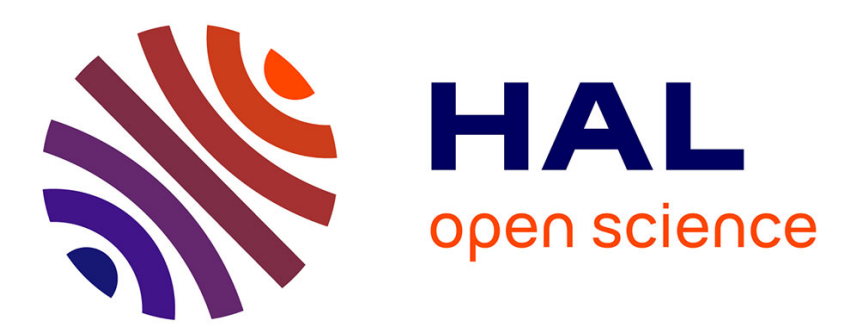

\title{
High-Gain Observer Design for a Class of Quasi-linear Integro-Differential Hyperbolic Systems - Application to an Epidemic Model
}

Constantinos Kitsos, Gildas Besancon, Christophe Prieur

\section{- To cite this version:}

Constantinos Kitsos, Gildas Besancon, Christophe Prieur. High-Gain Observer Design for a Class of Quasi-linear Integro-Differential Hyperbolic Systems - Application to an Epidemic Model. IEEE Transactions on Automatic Control, 2022, 67 (1), pp.292 - 303. 10.1109/TAC.2021.3063368 . hal03152772

\section{HAL Id: hal-03152772 \\ https://hal.science/hal-03152772}

Submitted on 25 Feb 2021

HAL is a multi-disciplinary open access archive for the deposit and dissemination of scientific research documents, whether they are published or not. The documents may come from teaching and research institutions in France or abroad, or from public or private research centers.
L'archive ouverte pluridisciplinaire HAL, est destinée au dépôt et à la diffusion de documents scientifiques de niveau recherche, publiés ou non, émanant des établissements d'enseignement et de recherche français ou étrangers, des laboratoires publics ou privés. 


\title{
High-Gain Observer Design for a Class of Quasi-linear Integro-Differential Hyperbolic Systems - Application to an Epidemic Model
}

\author{
Constantinos Kitsos, Gildas Besançon, and Christophe Prieur
}

\begin{abstract}
This work addresses the problem of High-Gain Observer design for a class of quasi-linear hyperbolic systems (with one characteristic velocity), possibly including nonlocal terms, making them systems of Partial Integro-Differential Equations. The design relies on distributed measurement of a part of the state vector. The observer is presented and discussed and the exponential stability in the $C^{1}$ spatial norm of the origin for the error system is fully established via Lyapunov-based analysis. Its use is illustrated via an application to an age-dependent SIR epidemic model.
\end{abstract}

Keywords: high-gain observers, PIDEs, nonlocal terms, quasilinear hyperbolic systems, Lyapunov analysis, $C^{1}$ exponential stability, SIR epidemic models.

\section{INTRODUCTION}

The classical high-gain observer design for finitedimensional nonlinear systems has been extensively studied in the literature and remains widely considered, see [21] and references therein. In short, it relies on a single tuning coefficient, to be chosen large enough so as to ensure exponential - and possibly arbitrarily fast - convergence. High-gain observers apply to a large class of cases corresponding to uniformly observable systems [14], [15]. In the recent paper [22], this approach was extended to a class of hyperbolic systems, for which first solutions to this "High-Gain Observer Design Problem" (H-GODP) have been proposed for a particular case of uniformly observable systems, written as an $n \times n$ quasi-linear hyperbolic system of balance laws and considering distributed measurements. In more recent works [23], [24] more general cases were considered, where $2 \times 2$ systems were written in an observable form with two distinct characteristic velocities, differentiating from [22], where it was needed to consider identical ones. There exist other studies on observer design for hyperbolic systems in the literature, mainly considering the full state vector on the boundaries as measurement. Amongst others, one can refer to [13] and [16] for the backstepping design, to [3] for a discretization approach, to [6] for direct infinite-dimension-based Lyapunov techniques (see also [4]), or to [28] for optimization methods. For semigroup-based methods, see [11], [7] and [31].

The present paper aims at providing a solution to the $\mathrm{H}$ GODP for a class of quasi-linear hyperbolic systems of balance laws, including nonlocal terms, i.e., systems of hyperbolic

C. Kitsos is with the LAAS-CNRS, Univ. of Toulouse, CNRS, Toulouse 31400, France and with the Université Grenoble Alpes, CNRS, Grenoble INP Institute of Engineering, GIPSA-lab, Grenoble 38402, France (email: ckitsos@laas.fr).

G. Besançon and C. Prieur are with the Université Grenoble Alpes, CNRS, Grenoble INP Institute of Engineering, GIPSA-lab, Grenoble 38402, France (emails: \{gildas.besancon, christophe.prieur\} @grenoble-inp.fr).
Partial Integro-Differential Equations (PIDEs), as an extension of [22]. The class of systems that we consider, written in an appropriate canonical form, can be found in various cases, like chemical reactors [5], where, by measuring the temperature of the reactor we would expect to estimate the concentrations of masses of chemicals, or some age-dependent epidemic models, where with the only measurement of the population that has recovered from a disease, the objective would be to estimate the remaining population groups arbitrarily fast. Stability and controllability of age-structured population models have been already studied (see [2, Chapter 1] and references therein, [32] and in general PIDEs or PDEs with nonlocal terms have been considered for instance in [9], [19], [12].

In the present approach, we consider systems having only one characteristic velocity, in the spirit of our former work of [22]: this results from a certain commutative property in the proposed Lyapunov stability analysis. Note that a similar constraint can be found in some studies of stability problems as in [2], [8] and in [30] (Proposition 2.1), and other works. This paper extends the first results of [22] in different ways: first, a larger class of systems (PIDE instead of PDE) is considered, i.e., hyperbolic systems including nonlocal terms; then, the conditions on the nonlinearities are weakened (to locally Lipschitz ones); in addition, a more general result of stability (here in the $C^{1}$, instead of the sup spatial norm) for the error equation is proven, with arguments inspired by [2] and [8] for instance; and finally, we illustrate the applicability of this approach to an epidemic model.

The paper is organized as follows. The solution to the H-GODP, the sufficient conditions and detailed proofs are provided in Section II, where Theorem 1 constitutes our main result. In Section III an application to an age-dependent SIRtype epidemic model is proposed. Conclusions and perspectives are discussed in Section IV.

Notation: For a given $w$ in $\mathbb{R}^{n},|w|$ denotes its usual Euclidean norm. For a given constant matrix $A$ in $\mathbb{R}^{n \times n}, A^{\top}$ denotes its transpose, $|A|:=\sup \{|A w|,|w|=1\}$ is its induced norm and $\operatorname{Sym}(A)=\frac{A+A^{\top}}{2}$ stands for its symmetric part. By eig $(A)$ we denote the minimum eigenvalue of a symmetric matrix $A$. By $I_{n \times n}$ we define the identity matrix of dimension $n$. For given $\xi:[0,+\infty) \times[0, L] \rightarrow \mathbb{R}^{n}$ and time $t \geq 0$ we use the notation $\xi(t)(x):=\xi(t, x)$, for all $x$ in $[0, L]$. For a continuous $\left(C^{0}\right)$ map $[0, L] \ni x \mapsto \xi(x) \in \mathbb{R}^{n}$ we adopt the notation $\|\xi\|_{\infty}:=\max \{|\xi(x)|, x \in[0, L]\}$. For a continuously differentiable $\left(C^{1}\right)$ map $[0, L] \ni x \mapsto \xi(x) \in \mathbb{R}^{n}$ we adopt the notation $\|\xi\|_{1}:=\|\xi\|_{\infty}+\left\|\xi_{x}\right\|_{\infty}$. For a mapping $f(\cdot, \cdot)$, we use the difference operator given by $\Delta_{\hat{\xi}}[f(\cdot, \mathcal{F})](\xi)(x):=$ 
$f(x, \mathcal{F}[\hat{\xi}](x))-f(x, \mathcal{F}[\xi](x))$, parametrized by $\hat{\xi}$, where $\mathcal{F}$ denotes any chosen operator acting on $\xi$. By $g^{\prime}$ we denote the usual derivative of a scalar function $g$. By $D_{u} f$ we denote the Jacobian of a differentiable mapping $\mathbb{R}^{n} \ni u \mapsto f(u) \in \mathbb{R}^{n}$. For a differentiable in its second argument mapping $f$, by $\left\langle D_{u} f(\cdot, \mathcal{F}[u]), h\right\rangle$ we denote its Fréchet derivative w.r.t. $u$ acting on $h$, where $\mathcal{F}$ is any Fréchet differentiable operator acting on functions $u$. Note that by the chain rule, $D_{u} f(\cdot, \mathcal{F}[u])=\left.D_{w} f(\cdot, w)\right|_{w=\mathcal{F}[u]} D_{u} \mathcal{F}[u]$, where $D_{u} \mathcal{F}[u]$ denotes the Fréchet derivative of $\mathcal{F}$. By $\mathcal{B}_{\delta}$ we denote the set $\mathcal{B}_{\delta}:=\left\{u \in C^{1}\left([0, L] ; \mathbb{R}^{n}\right):\|u\|_{1} \leq \delta\right\}$. By $\operatorname{sgn}(x)$ we denote the signum function $\operatorname{sgn}(x)=\frac{\mathrm{d}}{\mathrm{d} x}|x|$, when $x \neq$ 0 , with $\operatorname{sgn}(0)=0$.

\section{MAIN OBSERVER RESULT}

\section{A. Problem Statement and Requirements}

We are concerned with one-dimensional, first-order quasilinear hyperbolic system of balance laws, described by the following equations in a strip $\Pi:=[0,+\infty) \times[0, L] ; L>0$

$$
\begin{aligned}
& \xi_{t}(t, x)+\lambda\left(\xi_{1}(t, x)\right) \xi_{x}(t, x) \\
= & A \xi(t, x)+f(x, \mathcal{F}[\xi(t)](x))
\end{aligned}
$$

where $\left(\begin{array}{lll}\xi_{1} & \cdots & \xi_{n}\end{array}\right)^{\top}:=\xi$.

Consider also a distributed measurement, available in the output, of the form

$$
y(t, x)=C \xi(t, x)
$$

where $y:[0,+\infty) \times[0, L] \rightarrow \mathbb{R}$.

We assume that the involved constant matrices satisfy the following particular structures:

$$
A=\left(\begin{array}{ccccc}
0 & 1 & 0 & \cdots & 0 \\
& \ddots & \ddots & & \vdots \\
\vdots & & & & 1 \\
0 & \cdots & & & 0
\end{array}\right), C=\left(\begin{array}{llll}
1 & 0 & \cdots & 0
\end{array}\right)
$$

and the nonlinear balance terms are of the form

$$
f(\cdot, \mathcal{F}[\xi])=\left(\begin{array}{c}
f_{1}\left(\cdot, \mathcal{F}_{1}\left[\xi_{1}\right]\right) \\
f_{2}\left(\cdot, \mathcal{F}_{2}\left[\xi_{1}, \xi_{2}\right]\right) \\
\vdots \\
f_{n}\left(\cdot, \mathcal{F}_{n}\left[\xi_{1}, \ldots, \xi_{n}\right]\right)
\end{array}\right)
$$

From the previous equations, we observe that the system satisfies some triangular structure, which presents an analogy to the finite-dimensional case (see [21]).

Remark 1: Notice that if $f(\cdot, \mathcal{F}[\xi])$ reduces to $f(\xi)$ in (1a), implying that we have not any nonlocal terms, then we recover the more simple case of [22].

We assume the following regularity for the dynamics:

- the characteristic velocity $\lambda$ is continuously differentiable, i.e., $\lambda$ in $C^{1}(\mathbb{R} ; \mathbb{R})$ and, without loss of generality, $\lambda\left(\xi_{1}\right)>0$, for all $\xi_{1}$ in $\mathbb{R}$ (hyperbolicity condition).

- the nonlinear balance term $f$ is continuously differentiable, i.e., $f$ in $C^{1}\left([0, L] \times \mathbb{R}^{n} ; \mathbb{R}^{n}\right)$. As a result, for every $R>0$, there exists $L_{R}>0$, such that for every $w, \hat{w}$ in $\mathbb{R}^{n}$, with $|w|,|\hat{w}| \leq R$, $\max _{x \in[0, L]}|f(x, w)-f(x, \hat{w})| \leq L_{R}|w-\hat{w}|$. In addition, suppose that for every $R>0$, there exists $L_{R}^{\prime}>0$, such that for every $w, \hat{w}$ in $\mathbb{R}^{n}$, with $|w|,|\hat{w}| \leq R$, $\max _{x \in[0, L]}\left|D_{w} f(x, w)-D_{\hat{w}} f(x, \hat{w})\right| \leq L_{R}^{\prime}|w-\hat{w}|$. Furthermore, $\mathcal{F}: C^{1}\left([0, L] ; \mathbb{R}^{n}\right) \rightarrow C^{1}\left([0, L] ; \mathbb{R}^{n}\right)$ is a Fréchet differentiable mapping that can include terms $g_{1}(\xi)$, where $g_{1}$ in $C^{1}\left(\mathbb{R}^{n} ; \mathbb{R}^{n}\right)$ and also nonlinear nonlocal terms (integral terms of Volterra type) of the form $\int_{0}^{x} g_{2}(\xi(s)) \mathrm{d} s$, where $g_{2}$ in $C^{0}\left(\mathbb{R}^{n} ; \mathbb{R}^{n}\right)$. More explicitly, suppose that for every $R>0$, there exist $L_{1, R}, L_{2, R}, L_{3, R}, L_{1, R}^{\prime}, L_{2, R}^{\prime}, L_{3, R}^{\prime}>0$, such that for every $\xi, \hat{\xi}$ in $C^{0}\left([0, L] ; \mathbb{R}^{n}\right)$, with $\|\xi\|_{\infty},\|\hat{\xi}\|_{\infty} \leq R$, $\left|\Delta_{\hat{\xi}}[\mathcal{F}](\xi)(x)\right| \leq L_{1, R}|\xi(x)-\hat{\xi}(x)|+L_{2, R} \mid \xi(L)-$ $\hat{\xi}(L)\left|+L_{3, R} \int_{0}^{L}\right| \xi(s)-\hat{\xi}(s) \mid \mathrm{d} s$, and $\left|\Delta_{\hat{\xi}} D_{\xi}[\mathcal{F}](\xi)(x)\right| \leq$ $L_{1, R}^{\prime}|\xi(x)-\hat{\xi}(x)|+L_{2, R}^{\prime}|\xi(L)-\hat{\xi}(L)|+L_{3, R}^{\prime} \int_{0}^{L} \mid \xi(s)-$ $\hat{\xi}(s) \mid \mathrm{d} s$, for all $x$ in $[0, L]$.

We further consider initial and boundary conditions of the following general form

$$
\begin{aligned}
\xi(0, x) & =\xi^{0}(x), x \in[0, L] \\
\xi(t, 0) & =\mathcal{H}(\xi(t, L)), t \in[0,+\infty)
\end{aligned}
$$

where we assume the following regularity:

- initial condition $\xi^{0}$ is continuously differentiable and satisfies zero-order and one-order compatibility conditions (see [2, App. B] for precise definition of compatibility conditions). The mapping $\mathcal{H}$ is continuously differentiable, i.e., $\mathcal{H}$ in $C^{1}\left(\mathbb{R}^{n} ; \mathbb{R}^{n}\right)$. More explicitly, suppose that for every $R>0$, there exist $L_{4, R}, L_{4, R}^{\prime}>0$, such that for every $\xi, \hat{\xi}$ in $\mathbb{R}^{n}$, with $|\xi|,|\hat{\xi}| \leq R,\left|\Delta_{\hat{\xi}}[\mathcal{H}](\xi)(L)\right| \leq L_{4, R}|\xi(L)-\hat{\xi}(L)|$, and $\left|\Delta_{\hat{\xi}}\left[D_{\xi} \mathcal{H}\right](\xi)(L)\right| \leq L_{4, R}^{\prime}|\xi(L)-\hat{\xi}(L)|$.

Remark 2: The specific type of input/output relation (2b) on boundary conditions stands as a sufficient condition for the well-posedness of the observer. This will be shown in the sequel. Also, the main result of the present paper would remain unchanged if we considered the case where boundary conditions satisfy the relation $\xi(t, 0)=h(t, \xi(t, L))$, where $h(\cdot, \cdot)$ is a $C^{1}$ map. This case would require only slight modifications.

The problem that we address in this work is stated in the following definition.

Definition 1: (H-GODP) The High-Gain Observer Design Problem is solvable for a system given by (1a), (2) with output (1b) and solutions existing in $\Pi$, if there exists a well-posed observer system with solutions in $\Pi$, which estimates the state of (1a) with a convergence speed that can be arbitrarily tuned via a single parameter (high gain).

The assumption that follows is essential to assert the wellposedness of our system, along with an observer design requirement of forward completeness. Furthermore, it imposes global boundedeness of the classical solutions in the $C^{1}$-norm, which is essential in the design of our nonlinear observer. The latter requirement is due to the quasi-linearity of the system (the dependence of $\lambda$ on state $\xi$ ) and can be dropped for the case of semilinear systems, but then a stronger assumption on the nonlinear source terms would be imposed in its place. For 
more detailed presentation of the nature of this assumption, the reader can refer to [2], [26] and references therein, where sufficient conditions for the well posedness and existence of classical solutions for quasi-linear hyperbolic systems of balance laws are given.

Assumption 1: Consider a set $\mathcal{M} \subset C^{1}([0, L] ; \mathbb{R})$ nonempty and bounded, consisting of functions satisfying zero-order and one-order compatibility conditions for problem (1a)-(2). Then for any initial condition $\xi^{0}$ in $\mathcal{M}$, problem (1a)-(2) admits a unique classical solution in $C^{1}\left([0,+\infty) \times[0, L] ; \mathbb{R}^{n}\right)$. Moreover, there exists $\delta>0$, such that for all $\xi^{0}$ in $\mathcal{M},\|\xi(t, \cdot)\|_{1} \leq \delta$, for all $t$ in $[0,+\infty)$.

Define now a $C^{1}$ vector-valued function $\mathbb{R}^{n} \ni \zeta \mapsto s_{\delta}(\zeta)=$ $\left(s_{\delta}^{1}\left(\zeta_{1}\right), \cdots, s_{\delta}^{n}\left(\zeta_{n}\right)\right)$, parametrized by $\delta$, and satisfying the following properties.

For every $\delta>0$ and $v, w$ in $\mathbb{R}^{n}$, such that $|w| \leq \delta$, there exists $\omega_{\delta}>0$, such that the following inequalities are satisfied

$$
\begin{aligned}
\left|s_{\delta}(v)-w\right| & \leq \omega_{\delta}|v-w| \\
\left|D_{v} s_{\delta}(v)-D_{w} s_{\delta}(w)\right| & \leq \omega_{\delta}^{\prime}|v-w| .
\end{aligned}
$$

Moreover, there exists $m_{\delta}>0$, such that for every $v$ in $\mathbb{R}^{n}$,

$$
\left|s_{\delta}(v)\right|,\left|D_{v} s_{\delta}(v)\right| \leq m_{\delta}
$$

Note that a saturation-like function of the form

$$
s_{\delta}^{i}\left(\zeta_{i}\right)= \begin{cases}\zeta_{i}, & \left|\zeta_{i}\right| \leq \delta \\ \operatorname{sgn}\left(\zeta_{i}\right)\left(\left(\left|\zeta_{i}\right|-\delta\right) e^{-\left|\zeta_{i}\right|+\delta}+\delta\right), & \left|\zeta_{i}\right|>\delta\end{cases}
$$

satisfies (3) with $\omega_{\delta}=m_{\delta}=\sqrt{n} \max \left\{e^{-1}+\delta, 1\right\}, \omega_{\delta}^{\prime}=$ $\sqrt{n} e^{-3}$.

We are now in a position to introduce our candidate observer dynamics and its boundary conditions for system (1)-(2). Define, first, a diagonal matrix $\Theta$ by

$$
\Theta:=\operatorname{diag}\left\{\theta, \theta^{2}, \ldots, \theta^{n}\right\},
$$

where $\theta>1$ is the candidate high-gain constant of the observer, which will be selected precisely later. Consider, also, a vector gain $K$ in $\mathbb{R}^{n}$, such that $A+K C$ is Hurwitz (we can always find such a $K$, due to the observability of the pair $(A, C)$ ). Note that for such a $K$, one can find a symmetric and positive definite $n \times n$ matrix $P$ satisfying a quadratic Lyapunov equation of the following form

$$
2 \operatorname{Sym}(P(A+K C))=-I_{n \times n} .
$$

Let us remark that $P$ satisfying (6) cannot be diagonal, since matrix $A$ fails by its definition to be a diagonally stabilizable matrix. $P$ will be used as the Lyapunov matrix in the Lyapunov functional used in the proof of the observer convergence. However, in stability analysis of general hyperbolic systems, see for instance [2], the chosen Lyapunov functionals are diagonal, in order to commute with the matrix of the characteristic velocities. In the present case, we assume only one velocity and, thus, we do not need that $P$ is diagonal.
With the previous assumptions, the observer that will solve the H-GODP can be given by the following equations on $\Pi$

$$
\begin{gathered}
\hat{\xi}_{t}(t, x)+\lambda(y(t, x)) \hat{\xi}_{x}(t, x)=A \hat{\xi}(t, x) \\
-\Theta K(y(t, x)-C \hat{\xi}(t, x))+f\left(x, \mathcal{F}\left[s_{\delta}(\hat{\xi}(t))\right](x)\right) \\
\hat{\xi}(t, 0)=\mathcal{H}\left(s_{\delta}(\hat{\xi}(t, L))\right) .
\end{gathered}
$$

We can easily deduce some difference inequalities for the dynamics, to be invoked later, as a direct consequence of the regularity assumptions and properties (3) of $s_{\delta}(\cdot)$. Precisely, for any $\delta>0$, there exist constants $L_{1, \delta}, L_{2, \delta}, L_{3, \delta}, L_{4, \delta}, L_{1, \delta}^{\prime}, L_{2, \delta}^{\prime}, L_{3, \delta}^{\prime}, L_{4, \delta}^{\prime}>0$ depending on constants $\omega_{\delta}, \omega_{\delta}^{\prime}$, and $m_{\delta}$, such that for every $\xi, \hat{\xi}$ in $C^{0}\left([0, L] ; \mathbb{R}^{n}\right)$, with $\|\xi\|_{\infty} \leq \delta$, the following inequalities are satisfied for all $x$ in $[0, L]$ :

$$
\begin{aligned}
& \left|\Delta_{s_{\delta}(\hat{\xi})}[f(\cdot, \mathcal{F})](\xi)(x)\right| \\
& \leq L_{1, \delta}|\hat{\xi}(x)-\xi(x)|+L_{2, \delta}|\hat{\xi}(L)-\xi(L)| \\
& +L_{3, \delta} \int_{0}^{L}|\hat{\xi}(s)-\xi(s)| \mathrm{d} s, \\
& \left|\Delta_{s_{\delta}(\hat{\xi})}[\mathcal{H}](\xi)(L)\right| \leq L_{4, \delta}|\hat{\xi}(L)-\xi(L)|, \\
& \left|\Delta_{s_{\delta}(\hat{\xi})}\left[D_{\xi} f(\cdot, \mathcal{F})\right](\xi)(x)\right| \\
& \leq L_{1, \delta}^{\prime}|\hat{\xi}(x)-\xi(x)|+L_{2, \delta}^{\prime}|\hat{\xi}(L)-\xi(L)| \\
& +L_{3, \delta}^{\prime} \int_{0}^{L}|\hat{\xi}(s)-\xi(s)| \mathrm{d} s, \\
& \left|\Delta_{s_{\delta}(\hat{\xi})}\left[D_{\xi} \mathcal{H}\right](\xi)(L)\right| \leq L_{4, \delta}^{\prime}|\hat{\xi}(L)-\xi(L)| .
\end{aligned}
$$

Remark 3: Assumption 1 guarantees the existence of a preassigned ball in $C^{1}$ in which system's solutions take values. This, in conjunction with the regularity assumptions, would allow us to avoid globally Lipschitz assumption for the nonlinear dynamics of our system, which in general constitutes a natural assumption in classical high-gain observer designs for finite dimensions. To avoid such a restricting assumption, we plug function $s_{\delta}$ in observer dynamics (7). Exploiting this function guarantees that observer system's dynamics are globally Lipschitz. We note also that, although it is not described explicitly by the observer's equations, we avoid injecting $s_{\delta}$ in potential linear terms included in $f$ and $\mathcal{H}$. In that case, we set $s_{\delta}(\hat{\xi})=\hat{\xi}$, since linear terms are globally Lipschitz.

The following lemma guarantees the existence of unique global classical solutions for the candidate observer. The proof of the lemma follows from classical arguments and the fact that nonlinearities in observer system are globally Lipschitz.

Lemma 1: (Existence/Uniqueness of Global Classical Solutions to the Observer System) Under the regularity assumptions for the dynamics and for any $y$ in $C^{1}([0,+\infty) \times[0, L] ; \mathbb{R})$, the problem described by (7), with initial condition $\hat{\xi}^{0}(x):=\hat{\xi}(0, x)$, for all $x$ in $[0, L]$, satisfying zero-order and one-order compatibility conditions, admits a unique classical solution in $\Pi$, i.e., there exists a unique solution $\hat{\xi}$ in $C^{1}\left([0,+\infty) \times[0, L] ; \mathbb{R}^{n}\right)$ to $(7)$.

Proof: See Appendix A for a detailed proof.

We are now in a position to present our main result on the solvability of the H-GODP. 
Theorem 1: (Observer Convergence) Consider system (1a), (2), defined on $\Pi$ with output (1b) and suppose that Assumption 1 holds for initial condition $\xi^{0}$ in $\mathcal{M}$. Let also $K$ in $\mathbb{R}^{n}$, chosen in such a way that $A+K C$ is Hurwitz. Then, the $H$ GODP for system (1a) - (2) is solvable by system (7) for $\theta>1$ as a high gain and initial condition $\hat{\xi}^{0}$ in $C^{1}\left([0, L] ; \mathbb{R}^{n}\right)$, with $\hat{\xi}^{0}(x)=\hat{\xi}(0, x)$ satisfying zero-order and one-order compatibility conditions. This is a high-gain observer for $\xi$, in the sense that for $\theta$ large enough it admits a unique classical solution in $\Pi$ on the one hand, providing an estimate for the state of system (1a) - (2) on the other hand. More precisely, for every $\kappa>0$, there exists $\theta_{0} \geq 1$, such that for every $\theta>\theta_{0}$, the following inequality holds

$$
\|\hat{\xi}(t, \cdot)-\xi(t, \cdot)\|_{1} \leq \ell e^{-\kappa t}\left\|\hat{\xi}^{0}(\cdot)-\xi^{0}(\cdot)\right\|_{1}, \forall t \geq 0
$$

for some $\ell>1$, polynomial in $\theta$.

This theorem states that for system (1a) - (2) with output (1b) we have a high-gain observer design providing an estimate of its full state, with a convergence rate adjustable via $\theta$. The well posedness results from Lemma 1 . The convergence result is established in the next subsection.

\section{B. Observer Convergence Proof}

This subsection is dedicated to the proof of Theorem 1.

Consider the observer error $e:=\hat{\xi}-\xi$ which satisfies the following equations

$$
\begin{aligned}
& e_{t}(t, x)+ \lambda(y(t, x)) e_{x}(t, x)=(A+\Theta K C) e(t, x) \\
&+\Delta_{s_{\delta}(\hat{\xi}(t))}[f(\cdot, \mathcal{F})](\xi(t))(x), \\
& e(t, 0)=\Delta_{s_{\delta}(\hat{\xi}(t))}[\mathcal{H}](\xi(t))(L) .
\end{aligned}
$$

We now define the linearly transformed error by $\varepsilon:=\Theta^{-1} e$ and we derive the following hyperbolic equations for $\varepsilon$ on $\Pi$

$$
\begin{aligned}
& \varepsilon_{t}(t, x)+\lambda(y(t, x)) \varepsilon_{x}(t, x)=\theta(A+K C) \varepsilon(t, x) \\
&+\Theta^{-1} \Delta_{s_{\delta}}(\hat{\xi}(t))[f(\cdot, \mathcal{F})](\xi(t))(x), \\
& \varepsilon(t, 0)=\Theta^{-1} \Delta_{s_{\delta}(\hat{\xi}(t))}[\mathcal{H}](\xi(t))(L) .
\end{aligned}
$$

At this point, let us introduce an operator $\mathcal{K}$ : $C^{1}\left([0, L] ; \mathbb{R}^{n}\right) \rightarrow C^{0}\left([0, L] ; \mathbb{R}^{n \times n}\right)$ defined by

$$
\begin{aligned}
\mathcal{K}[\xi]:= & \lambda^{\prime}(C \xi)(\lambda(C \xi))^{-1} I_{n \times n} C \\
& \times\left(-\lambda(C \xi) \frac{\partial}{\partial x} \xi+A \xi+f(\cdot, \mathcal{F}[\xi])\right) .
\end{aligned}
$$

Define also $\mathcal{K}_{1}^{\hat{\xi}}: C^{1}\left([0, L] ; \mathbb{R}^{n}\right) \rightarrow C^{0}\left([0, L] ; \mathbb{R}^{n}\right)$, parametrized by $\hat{\xi} \in C^{0}\left([0, L] ; \mathbb{R}^{n}\right)$ and given by

$$
\begin{aligned}
\mathcal{K}_{1}^{\hat{\xi}}[\xi]:= & -\mathcal{K}[\xi] \Theta^{-1} \Delta_{s_{\delta}(\hat{\xi})}[f(\cdot, \mathcal{F})](\xi) \\
& +\Theta^{-1}\left(\left\langle\Delta_{s_{\delta}(\hat{\xi})}\left[D_{\xi} f(\cdot, \mathcal{F})\right](\xi), D_{\hat{\xi}} s_{\delta}(\hat{\xi}) \xi_{t}\right\rangle\right. \\
& \left.+\left\langle\left. D_{u} f(\cdot, \mathcal{F}[u])\right|_{u=s_{\delta}(\hat{\xi})}, \Delta_{\hat{\xi}}\left[D_{\xi} s_{\delta}\right](\xi) \xi_{t}\right\rangle\right)
\end{aligned}
$$

Next, by temporarily assuming that $\varepsilon$ is of class $C^{2}$, we derive the following hyperbolic equations for $\varepsilon_{t}$, where we have also substituted the expression of $\varepsilon_{x}$ derived from (12),

$$
\begin{aligned}
& \varepsilon_{t t}(t, x)+\lambda(y(t, x)) \varepsilon_{t x}(t, x)=\mathcal{K}[\xi(t)](x) \varepsilon_{t}(t, x) \\
& +\Theta^{-1}\left\langle\left. D_{u} f(\cdot, \mathcal{F}[u])\right|_{u=s_{\delta}(\xi(t))},\right. \\
& \left.D_{\hat{\xi}} s_{\delta}(\hat{\xi}(t)) \Theta \varepsilon_{t}(t)\right\rangle(x)+\theta(A+K C) \varepsilon_{t}(t, x) \\
& -\theta \mathcal{K}[\xi(t)](x)(A+K C) \varepsilon(t, x)+\mathcal{K}_{1}^{\hat{\xi}(t)}[\xi(t)](x),
\end{aligned}
$$

$$
\begin{aligned}
& \varepsilon_{t}(t, 0)=\Theta^{-1}\left(\Delta_{s_{\delta}(\hat{\xi}(t))}\left[D_{\xi} \mathcal{H}\right](\xi(t))(L) D_{\hat{\xi}} s_{\delta}(\hat{\xi}(t, L))\right. \\
& \times \xi_{t}(t, L)+\left.D_{u} \mathcal{H}(u)\right|_{u=s_{\delta}(\hat{\xi}(t))}(L) D_{\hat{\xi}} s_{\delta}(\hat{\xi}(t, L)) \Theta \varepsilon_{t}(t, L) \\
& \left.+D_{\xi} \mathcal{H}(\xi(t, L)) \Delta_{\hat{\xi}(t)}\left[D_{\xi} s_{\delta}\right](\xi(t))(L) \xi_{t}(t, L)\right) .
\end{aligned}
$$

Remark 4: Due to cumbersome notation in the previous relations, we provide an example to make the calculations of the Fréchet derivative more clear. Let, for instance, $f(x, \mathcal{F}[u](x))=g(x) g_{1}(u(x))+g_{2}(u(L))+\int_{0}^{x} g_{3}(u(s)) \mathrm{d} s$. Then, $\left\langle D_{u} f(\cdot, \mathcal{F}[u]), u_{t}\right\rangle(x)=g(x) D_{u} g_{1}(u)(x) u_{t}(x)$ $+D_{u} g_{2}(u)(L) u_{t}(L)+\int_{0}^{x} D_{u} g_{3}(u)(s) u_{t}(s) \mathrm{d} s$.

Now, notice that whenever $\xi$ in $\mathcal{B}_{\delta}$, due to continuity of $\lambda(\cdot)$, the fact that $\lambda\left(\xi_{1}\right)>0$, for all $\xi_{1}$ in $\mathbb{R}$ and the fact that $\|\xi\|_{\infty} \leq$ $\delta$, the quantities $\sup _{\xi \in \mathcal{B}_{\delta}}(\lambda(C \xi)), \inf _{\xi \in \mathcal{B}_{\delta}}(\lambda(C \xi))$ are positive and finite. In addition, whenever $\xi$ in $\mathcal{B}_{\delta}$ and invoking the hyperbolic dynamics (1a), we can easily calculate constant $\delta_{1}>0$, dependent on $\delta$, such that

$$
\left\|\xi_{t}\right\|_{\infty}=\left\|-\lambda\left(\xi_{1}\right) \xi_{x}+A \xi+f(\cdot, \mathcal{F}[\xi])\right\|_{\infty} \leq \delta_{1} .
$$

By combining the above arguments, the fact that $\theta>1$, (18), continuity of the involved mappings, and inequalities (8), we can easily calculate positive constants $\gamma_{i, \delta}, i=1, \ldots, 6$, such that whenever $\xi$ in $\mathcal{B}_{\delta}, \hat{\xi}$ in $C^{0}\left([0, L] ; \mathbb{R}^{n}\right)$, the following inequalities are satisfied for all $x$ in $[0, L]$

$$
\begin{gathered}
\left|\Theta^{-1}\left\langle\left. D_{u} f(\cdot, \mathcal{F}[u])\right|_{u=s_{\delta}(\hat{\xi})}, D_{\hat{\xi}} s_{\delta}(\hat{\xi}) \Theta \varepsilon_{t}\right\rangle(x)\right| \\
\leq \gamma_{1, \delta}\left(\left|\varepsilon_{t}(x)\right|+\left|\varepsilon_{t}(L)\right|+\int_{0}^{L}\left|\varepsilon_{t}(s)\right| \mathrm{d} s\right) \\
\left|\Theta^{-1} \Delta_{s_{\delta}(\hat{\xi})}[f(\cdot, \mathcal{F})](\xi)(x)\right| \leq \gamma_{2, \delta}(|\varepsilon(x)|+|\varepsilon(L)| \\
\left.\quad+\int_{0}^{L}|\varepsilon(s)| \mathrm{d} s\right) \\
\left|\Theta^{-1} \Delta_{s_{\delta}(\hat{\xi})}[\mathcal{H}](\xi)(L)\right| \leq \gamma_{3, \delta} \theta^{n-1}|\varepsilon(L)|, \\
\quad+\int_{0}^{-1} \Delta_{s_{\delta}(\hat{\xi})}\left[D_{\xi} f(\cdot, \mathcal{F})\right](\xi)(x) \mid \leq \gamma_{4, \delta}(|\varepsilon(x)|+|\varepsilon(L)| \\
\left|\Theta^{-1} D_{u} \mathcal{H}[u]\right|_{u=s_{\delta}(\hat{\xi})}(L) D_{\hat{\xi}} s_{\delta}(\hat{\xi}(L)) \Theta \mid \leq \gamma_{5, \delta} \theta^{n-1}, \\
\mid \Theta^{-1}\left(\Delta_{s_{\delta}(\hat{\xi})}\left[D_{\xi} \mathcal{H}\right](\xi)(L) D_{\hat{\xi}} s_{\delta}(\hat{\xi})\right. \\
\left.+D_{\xi} \mathcal{H}(\xi(L)) \Delta_{\hat{\xi}}\left[D_{\xi} s_{\delta}\right](\xi)(L)\right)\left|\leq \gamma_{6, \delta} \theta^{n-1}\right| \varepsilon(L) \mid .
\end{gathered}
$$


Define also constants

$$
\begin{aligned}
\gamma_{7, \delta} & :=\sup _{\xi \in \mathcal{B}_{\delta}}\left|\lambda^{\prime}(C \xi)\right|, \gamma_{8, \delta}:=\inf _{\xi \in \mathcal{B}_{\delta}} \lambda(C \xi), \\
\gamma_{9, \delta} & :=\sup _{\xi \in \mathcal{B}_{\delta}} \lambda(C \xi),
\end{aligned}
$$

and derive the following bounds for all $x$ in $[0, L]$

$$
\begin{aligned}
|\mathcal{K}[\xi](x)| & \leq \gamma_{10, \delta}:=\frac{\delta_{1} \gamma_{7, \delta}}{\gamma_{8, \delta}}, \\
\left|\mathcal{K}_{1}^{\hat{\xi}}[\xi](x)\right| & \leq \gamma_{11, \delta}\left(|\varepsilon(x)|+|\varepsilon(L)|+\int_{0}^{L}|\varepsilon(s)| \mathrm{d} s\right) \\
\gamma_{11, \delta} & :=\gamma_{10, \delta} \gamma_{2, \delta}+\delta_{1} \gamma_{4, \delta} .
\end{aligned}
$$

Remark 5: Note here that all constants $\gamma_{1, \delta}, \ldots, \gamma_{11, \delta}$ are calculated by taking bounds of the involved mappings on $\mathcal{B}_{\delta}$ and depend on the global bound $\delta$ of the system's trajectories (and subsequently on $\delta_{1}$ in (18)), constants in (8), the order of the system $n$, and are independent of the observer gain $\theta$. More explicitly, $\gamma_{1, \delta}, \gamma_{2, \delta}, \gamma_{4, \delta}$, and $\gamma_{11, \delta}$ being independent of $\theta$ is a direct consequence of the assumed triangularity of the involved mappings, similarly as in the classical high-gain observer designs [15]. This property turns out to be sufficient for the solvability of the H-GODP. More precisely, in the sequel, while bounding the Lyapunov time-derivative from above, the independence of these parameters on $\theta$ shall not add positive terms with linear (or higher-order) dependency on $\theta$. On the other hand, negative terms will appear depending linearly on $\theta$ as a direct consequence of the assumed observability of the pair $(A, C)$. This will render the negativity of the Lyapunov time-derivative feasible, as this will be more clear in the forthcoming Lyapunov analysis.

To prove the exponential stability of the solution to the error system at the origin, we need to define a Lyapunov functional $\mathcal{W}_{p}: C^{1}\left([0, L] ; \mathbb{R}^{n}\right) \rightarrow \mathbb{R}$ by

$$
\mathcal{W}_{p}[\varepsilon]:=\left(\int_{0}^{L} \pi(x) \exp \left(p \mu_{\theta, \delta} x\right) \mathcal{G}_{p}[\varepsilon](x) \mathrm{d} x\right)^{1 / p}
$$

where $\mathcal{G}_{p}: C^{1}\left([0, L] ; \mathbb{R}^{n}\right) \rightarrow \mathbb{R}$ is given by

$$
\mathcal{G}_{p}[\varepsilon]:=\left(\varepsilon^{\top} P \varepsilon+\rho_{0} \varepsilon_{t}^{\top} P \varepsilon_{t}\right)^{p}
$$

and $\rho_{0}$ in $(0,1)$ is a constant (to be chosen appropriately), $p$ in $\mathbb{N}, P$ in $\mathbb{R}^{n \times n}$ is positive definite symmetric satisfying (6), $\pi:[0, L] \rightarrow \mathbb{R}$ is given by

$$
\pi(x):=(\bar{\pi}-1) \frac{x}{L}+1 ; \bar{\pi}:=\frac{\sup _{\xi \in \mathcal{B}_{\delta}} \lambda(C \xi)}{\inf _{\xi \in \mathcal{B}_{\delta}} \lambda(C \xi)}
$$

and constant $\mu_{\theta, \delta}$ is given by

$$
\mu_{\theta, \delta}:=\frac{1}{L} \ln \left(\mu_{\delta} \theta^{2 n-2}\right)
$$

where

$$
\mu_{\delta}:=\frac{|P|}{\underline{\operatorname{eig}(P)}} \max \left\{\gamma_{3, \delta}^{2}, \gamma_{5, \delta}^{2}, \gamma_{6, \delta}^{2} \delta_{1}^{2}, \gamma_{5, \delta} \gamma_{3, \delta} \delta_{1}\right\} .
$$

Note here that, by its definition, $\pi$ is bounded as follows

$$
1 \leq \pi(x) \leq \bar{\pi}, \forall x \in[0, L]
$$

Let us also define functional $\mathcal{V}: C^{1}\left([0, L] ; \mathbb{R}^{n}\right) \rightarrow \mathbb{R}$ by

$$
\mathcal{V}[\varepsilon]:=\left\|\exp \left(\mu_{\theta, \delta^{*}}\right) \varepsilon^{\top} P \varepsilon\right\|_{\infty}+\left\|\rho_{0} \exp \left(\mu_{\theta, \delta^{*}}\right) \varepsilon_{t}^{\top} P \varepsilon_{t}\right\|_{\infty} .
$$

The idea is to let $p \rightarrow \infty$ in the end, to approximate $V$ by $W_{p}$. This convergence property is presented in details in Appendix B. By invoking Lemma 1 and Assumption 1, which establish global unique classical solutions for both observer system (7) and system (1a), (2) respectively, we are now in a position to define $G_{p}, W_{p}, V:[0,+\infty) \rightarrow \mathbb{R}$ by

$$
G_{p}(t):=\mathcal{G}_{p}[\varepsilon](t), W_{p}(t):=\mathcal{W}_{p}[\varepsilon](t), V(t):=\mathcal{V}[\varepsilon](t), \forall t \geq 0 .
$$

Calculating the time-derivative $\dot{W}_{p}$ along the classical solutions to (12) - (13), (16) - (17), we get

$$
\begin{gathered}
\dot{W}_{p}=\frac{1}{p} W_{p}^{1-p} \int_{0}^{L} p \pi(x) \exp \left(p \mu_{\theta, \delta} x\right) G_{p-1}(x) \\
\times\left(\varepsilon_{t}(x)^{\top} P \varepsilon(x)+\varepsilon^{\top}(x) P \varepsilon_{t}(x)+\rho_{0} \varepsilon_{t t}^{\top}(x) P \varepsilon_{t}(x)\right. \\
\left.+\rho_{0} \varepsilon_{t}(x)^{\top} P \varepsilon_{t t}(x)\right) \mathrm{d} x .
\end{gathered}
$$

After substituting the dynamical equations (12) and (16) into the above equation and performing integration by parts, $\dot{W}_{p}$ can be written in the following form

$$
\dot{W}_{p}=W_{p}^{1-p}\left(\frac{1}{p} T_{1, p}+\frac{1}{p} T_{2, p}+T_{3, p}+T_{4, p}+T_{5, p}\right)
$$

where

$$
\begin{gathered}
T_{1, p}:=-\pi(L) \lambda(y(L)) \exp \left(p \mu_{\theta, \delta} L\right) G_{p}(L) \\
+\pi(0) \lambda(y(0)) G_{p}(0), \\
\left.T_{2, p}:=\int_{0}^{L} \partial_{x}\left[\pi(x) \exp \left(p \mu_{\theta, \delta} x\right) \lambda(y(x))\right] G_{p}(x) \mathrm{d} x, \quad \text { (29) }\right) \\
T_{3, p}:=2 \int_{0}^{L} \pi(x) \exp \left(p \mu_{\theta, \delta} x\right) G_{p-1}(x)\left[\varepsilon^{\top}(x) P \Theta^{-1}\right. \\
\times \Delta_{s_{\delta}(\hat{\xi})}[f(\cdot, \mathcal{F})](\xi)(x)+\rho_{0} \varepsilon_{t}^{\top}(x) P \mathcal{K}_{1}^{\hat{\xi}}[\xi](x) \\
\left.+\rho_{0} \varepsilon_{t}^{\top}(x) \operatorname{Sym}(P \mathcal{K}[\xi](x)) \varepsilon_{t}(x)\right] \mathrm{d} x, \\
T_{4, p}:=2 \int_{0}^{L} \pi(x) \exp \left(p \mu_{\theta, \delta} x\right) G_{p-1}(x) \rho_{0} \varepsilon_{t}(x)^{\top} P \Theta^{-1} \\
\times\left\langle\left. D_{u} f(\cdot, \mathcal{F}[u])\right|_{u=s \delta}(\hat{\xi}), D_{\hat{\xi}} s_{\delta}(\hat{\xi}) \Theta \varepsilon_{t}\right\rangle(x) \mathrm{d} x, \quad(29 \mathrm{~d}) \\
T_{5, p}:=\theta \int_{0}^{L} \pi(x) \exp \left(p \mu_{\theta, \delta} x\right) G_{p-1}(x) \\
\times\left[2 \varepsilon ^ { \top } \operatorname { S y m } \left(P(A+K C) \varepsilon+2 \rho_{0} \varepsilon_{t}^{\top} \operatorname{Sym}(P(A+K C)) \varepsilon_{t}\right.\right. \\
\left.-\rho_{0} \varepsilon_{t}^{\top} P \mathcal{K}[\xi](A+K C) \varepsilon-\rho_{0} \varepsilon^{\top}(A+K C)^{\top} \mathcal{K}^{\top}[\xi] P \varepsilon_{t}\right] \mathrm{d} x .
\end{gathered}
$$

After substituting boundary equations (13) and (17) in $T_{1, p}$ and by virtue of (8) and (22), using the previously calculated bounds and trivial inequalities, we can easily obtain the following inequality

$$
T_{1, p} \leq \sup _{\xi \in \mathcal{B}_{\delta}}(\lambda(C \xi)) G_{p}(L)\left(-\exp \left(p \mu_{\theta, \delta} L\right)+\left(\theta^{2 n-2} \mu_{\delta}\right)^{p}\right)
$$

and, subsequently, by (23a)

$$
T_{1, p} \leq 0
$$


By the fact that $\pi(x) \geq 1$, for all $x$ in $[0, L]$, we can derive the following bound for $T_{2, p}$

$$
T_{2, p} \leq\left(\omega_{1, \delta}+p\left|\mu_{\theta, \delta}\right| \omega_{2, \delta}\right) W_{p}^{p}
$$

where

$$
\omega_{1, \delta}:=\frac{|P| \delta \gamma_{7, \delta}}{\underline{\operatorname{eig}}(P)}, \quad \omega_{2, \delta}:=\frac{|P| \gamma_{9, \delta}}{\underline{\operatorname{eig}}(P)} .
$$

By exploiting (19)-(20), $T_{3, p}$ can be bounded as follows

$$
\begin{gathered}
T_{3, p} \leq 2 \int_{0}^{L} \pi(x) \exp \left(p \mu_{\theta, \delta} x\right) G_{p-1}(x)|P| \\
\times\left[\gamma_{2, \delta}|\varepsilon(x)|\left(|\varepsilon(x)|+|\varepsilon(L)|+\int_{0}^{x}|\varepsilon(s)| \mathrm{d} s\right)\right. \\
+\rho_{0} \gamma_{11, \delta}\left|\varepsilon_{t}(x)\right|\left(|\varepsilon(x)|+|\varepsilon(L)|+\int_{0}^{x}|\varepsilon(s)| \mathrm{d} s\right) \\
\left.\quad+\rho_{0} \gamma_{10, \delta}\left|\varepsilon_{t}(x)\right|^{2}\right] \mathrm{d} x \\
\times \int_{0}^{L} \pi(x) \exp \left(p \mu_{\theta, \delta} x\right) G_{p-1}(x) \frac{|P|}{\operatorname{eig}(P)} \\
+\int_{0}^{L} \pi(x) \exp \left(p \mu_{\theta, \delta} x\right) G_{p-1}(x) \frac{|P|}{\operatorname{eig}(P)} \\
\times\left(\gamma_{2, \delta}+\rho_{0} \gamma_{11, \delta}\right)\left(G_{1}(L)+\int_{0}^{x} G_{1}(s) \mathrm{d} s\right) \mathrm{d} x \\
\left.\leq \omega_{3, \delta} W_{p}^{p}+\omega_{4, \delta}\left\|\exp \left(p \mu_{\theta, \delta} \cdot\right) G_{1}(\cdot)\right\| \gamma_{11, \delta}, 3 \gamma_{11, \delta}^{p-1}+\gamma_{10, \delta}\right\} \\
\leq \omega_{3, \delta} W_{p}^{p}+\omega_{4, \delta} V W_{p-1}^{p-1},
\end{gathered}
$$

where

$$
\begin{aligned}
\omega_{3, \delta} & :=\frac{|P|}{\frac{\operatorname{eig}(P)}{|P|} \max \left\{4 \gamma_{2, \delta}+\rho_{0} \gamma_{11, \delta}, 3 \gamma_{11, \delta}+\gamma_{10, \delta}\right\}} \\
\omega_{4, \delta} & :=\frac{|P|}{\underline{\operatorname{eig}(P)}}\left(\gamma_{2, \delta}+\rho_{0} \gamma_{11, \delta}\right)(1+L) .
\end{aligned}
$$

Similarly, we can bound $T_{4, p}$ as follows

$$
T_{4, p} \leq \omega_{5, \delta} W_{p}^{p}+\omega_{6, \delta} V W_{p-1}^{p-1}
$$

where

$$
\omega_{5, \delta}:=2 \frac{|P|}{\underline{\operatorname{eig}}(P)} \gamma_{1, \delta}, \omega_{6, \delta}:=2 \frac{|P|}{\underline{\operatorname{eig}(P)}} \gamma_{1, \delta}(1+L) .
$$

The term $T_{5, p}$ of $\dot{W}_{p}$ can be rewritten in the following way

$$
\begin{aligned}
& T_{5, p}:=-\theta \int_{0}^{L} \pi(x) \exp \left(p \mu_{\theta, \delta} x\right) G_{p-1}(x)
\end{aligned}
$$

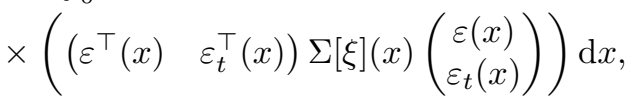

where, after utilizing (6), $\Sigma: \mathcal{B}_{\delta} \rightarrow C^{0}\left([0, L] ; \mathbb{R}^{2 n \times 2 n}\right)$ is given by

$\Sigma[\xi]:=\left(\begin{array}{cc}I_{n \times n} & -\rho_{0}(A+K C)^{\top} \mathcal{K}^{\top}[\xi] P \\ -\rho_{0} P \mathcal{K}[\xi](A+K C) & \rho_{0} I_{n \times n}\end{array}\right)$.

Now, we can easily verify (using Schur complement) that for all $w \in \mathbb{R}^{2 n} \backslash 0$, we have $\inf _{\xi \in \mathcal{B}_{\delta}} \frac{w^{\top} \Sigma[\xi] w}{|w|^{2}}>0$, if

$$
0<\rho_{0}<\min \left(\frac{1}{|P|^{2}|A+K C|^{2} \gamma_{10, \delta}^{2}}, 1\right) \text {. }
$$

It turns out that for every choice of matrices $P$ and $K$ satisfying (6), there always exists a $\rho_{0}$, such that (38) is satisfied and this fact renders $\Sigma$ positive. Consequently, for appropriate choice of $\rho_{0}$, there exists $\sigma_{\delta}>0$, such that

$$
T_{5, p} \leq-\theta \frac{\sigma_{\delta}}{|P|} W_{p}^{p}
$$

Combining (31), (32), (33), (35), and (39) with (28), we obtain

$$
\begin{aligned}
\dot{W}_{p} & \leq\left(-\theta \omega_{7, \delta}+\omega_{8, \delta} \ln (\theta)+\omega_{9, \delta}\right) W_{p} \\
& +\left(\omega_{4, \delta}+\omega_{6, \delta}\right) W_{p}^{1-p} W_{p-1}^{p-1} V,
\end{aligned}
$$

where $\omega_{7, \delta}:=\frac{\sigma_{\delta}}{|P|}, \omega_{8, \delta}:=\frac{\omega_{2, \delta}(2 n-2)}{L}, \omega_{9, \delta}:=\omega_{1, \delta}+\omega_{3, \delta}+$ $\frac{\omega_{2, \delta}}{L}\left|\ln \mu_{\delta}\right|$.

Now, using Hölder's inequality, one can obtain

$$
W_{p-1}^{p-1} \leq W_{p}^{p-1}\|\pi(\cdot)\|_{\infty}^{1 / p}
$$

Utilizing the above inequality, (40) gives

$$
\begin{gathered}
\dot{W}_{p} \leq\left(-\theta \omega_{7, \delta}+\omega_{8, \delta} \ln (\theta)+\omega_{9, \delta}\right) W_{p} \\
+\left(\omega_{4, \delta}+\omega_{6, \delta}\right) \bar{\pi}^{1 / p} V .
\end{gathered}
$$

We obtained the estimate (41) of $\dot{W}_{p}$ for $\varepsilon$ of class $C^{2}$, but, by invoking density arguments, the results remain valid with $\varepsilon$ only of class $C^{1}$ (see [8] for further details).

From (41), taking the limit as $p \rightarrow+\infty$ and using the fact that $\lim _{p \rightarrow+\infty} \bar{\pi}^{1 / p}=1$, we get in the distribution sense in $(0,+\infty)$,

$$
\dot{V}(t) \leq\left(-\theta \omega_{7, \delta}+\omega_{8, \delta} \ln (\theta)+\omega_{10, \delta}\right) V(t)
$$

where $\omega_{10, \delta}:=\omega_{4, \delta}+\omega_{6, \delta}+\omega_{9, \delta}$. For more details on how to obtain the above estimate, see Properties 1 and 2 in Appendix B.

Now, one can select the high gain $\theta$, such that

$$
\theta>\theta_{0}
$$

where $\theta_{0} \geq 1$ is such that

$$
-\theta \omega_{7, \delta}+\omega_{8, \delta} \ln (\theta)+\omega_{10, \delta} \leq-2 \kappa_{\delta}, \forall \theta>\theta_{0}
$$

for some $\kappa_{\delta}>0$. One can easily check that for any $\kappa_{\delta}>$ 0 , there always exists a $\theta_{0} \geq 1$, dependent on the involved constants, such that the previous inequality is satisfied.

Subsequently, (42) yields to the following differential inequality in the distribution sense in $(0,+\infty)$

$$
\dot{V}(t) \leq-2 \kappa_{\delta} V(t)
$$

and by the comparison lemma, we get

$$
V(t) \leq e^{-2 \kappa_{\delta} t} V(0), \forall t \geq 0 .
$$

Now, by the dynamics (12), in conjunction with (19), (20), we can obtain the following inequalities

$$
\begin{aligned}
& \left\|\varepsilon_{t}\right\|_{\infty} \geq \gamma_{8, \delta}\left\|\varepsilon_{x}\right\|_{\infty}-s_{\delta, \theta}\|\varepsilon\|_{\infty} \\
& \left\|\varepsilon_{t}\right\|_{\infty} \leq \gamma_{9, \delta}\left\|\varepsilon_{x}\right\|_{\infty}+s_{\delta, \theta}\|\varepsilon\|_{\infty}
\end{aligned}
$$


where $s_{\delta, \theta}:=\theta|A+K C|+\gamma_{2, \delta}(2+L)$. Invoking these inequalites, also (23a), estimate (46) and the following inequality,

$$
\begin{gathered}
\frac{\rho_{0}}{2} e^{\frac{\mu_{\theta, \delta}-\left|\mu_{\theta, \delta}\right|}{2} L} \underline{\operatorname{eig}}(P)\left(\|\varepsilon\|_{\infty}+\left\|\varepsilon_{t}\right\|_{\infty}\right)^{2} \leq V \\
\leq e^{\frac{\mu_{\theta, \delta}+\left|\mu_{\theta, \delta}\right|}{2}} L|P|\left(\|\varepsilon\|_{\infty}+\left\|\varepsilon_{t}\right\|_{\infty}\right)^{2}
\end{gathered}
$$

we obtain

$$
\|\varepsilon\|_{1} \leq \ell_{\delta, \theta} e^{-\kappa_{\delta} t}\left\|\varepsilon^{0}\right\|_{1}, \forall t \geq 0,
$$

where $\varepsilon^{0}(x):=\varepsilon(0, x)$ and

$$
\begin{aligned}
\ell_{\delta, \theta}:= & \sqrt{\frac{|P|}{\rho_{0} \underline{\operatorname{eig}(P)}}\left(\mu_{\delta}\right)^{\frac{1}{2 L}} \theta^{\frac{n-1}{L}}} \\
& \times \max \left\{s_{\delta, \theta}+1, \frac{1}{\gamma_{8, \delta}}\right\} \max \left\{1+2 s_{\delta, \theta}, 2 \gamma_{9, \delta}\right\} .
\end{aligned}
$$

By (47), we derive the following estimate, which holds for every $t \geq 0$

$$
\|\hat{\xi}(t, \cdot)-\xi(t, \cdot)\|_{1} \leq \bar{\ell}_{\delta, \theta} e^{-\kappa_{\delta} t}\left\|\hat{\xi}^{0}-\xi^{0}\right\|_{1},
$$

where $\bar{\ell}_{\delta, \theta}:=\theta^{n-1} \ell_{\delta, \theta}$.

Concluding, we solved the H-GODP for (1a)-(1b)-(2) by designing an exponential in the $C^{1}$-norm high-gain observer of adjustable convergence rate $\kappa_{\delta}$, dependent on the selection of $\theta$. The proof of Theorem 1 is complete.

Remark 6: Notice that the H-GODP is not solvable in case of boundary measurement, instead of internal measurement as in (1b). First, arbitrary convergence condition would not be fulfilled, since a boundary-based observer for hyperbolic systems would experience a limitation with respect to convergence speed. The rate of convergence is limited by a minimal observation time which depends on the size of the domain $L$ and the characteristic velocities in that case (see [27] for minimum time of observability due to transport phenomena, and [12] for comments on the convergence of boundary observers). Second, boundary measurement of the whole state vector would be required, instead for just the first state, in order to end up in some dissipativity in the boundary conditions of the error system (as in [6] for instance). In [10], a backstepping design is proposed for some kind of dual control problem via boundary control of only one state, but in our observer problem, because of the generality of our boundary conditions, we cannot achieve their dissipativity by one observation only, which would lead to stability of the observation error system (see [8] about linking dissipativity of boundary conditions with stability). In general, the problem of controllability and observability for cascade systems with reduced numbers of controls and observations, less than the number of states, is a hard problem, see for instance [1].

Remark 7: The observer design proposed in this work relies on a continuous measurement in the whole space, see (1b). This means that a direct application a priori needs the system to have such measurements. In addition, due to the absence of localized coupling between the nonlinear PDEs, as the coupling matrix $A$ is constant, it is hard to see how a possibly localized measurement in a part of the domain could be used in order to guarantee the existence of a damping term as $(A+K C)$ in (12), that would render the Lyapunov derivative negative. However, some improvement for practical implementation could be to consider an output of the form (1b), but with $C$ being substituted by $C(x):=c(x) C$, where $c(x)=\sum_{i=1}^{N} e^{-a_{i}\left(x-x_{i}\right)^{2}}$, with $a_{i}$ sufficiently large. This would correspond to a continuous measurement again, but that would have higher contribution around points $x_{i}, i=1, \ldots, N$ inside the domain and less contribution far from them. Then, the damping term would be $A+K(x) C(x)$, with $K(x)$ chosen such that $A+K(x) C(x)$ is Hurwitz. Such improvements will be a subject of future works.

\section{APPLICATION}

As an illustrative example, let us consider the case of an epidemic model. In the field of epidemiology, mathematical models are currently used to explain epidemic phenomena and to assess vaccination strategies through control mechanisms. For infectious diseases, a fundamental model was formulated by Kermack and McKendrick in 1927 [20]. This model classifies population into three groups: (i) the individuals who are uninfected and susceptible $(S)$ of catching the disease, (ii) the individuals who are infected $(I)$ by the concerned pathogen, (iii) the recovered $(R)$ individuals who have acquired a permanent immunity to the disease. In the case when the age of patients is taken into account, $S(t, x), I(t, x), R(t, x)$ represent the age distribution of the population of each group at time $t$. As a result, the integral from $x_{1}$ to $x_{2}$ of $S, I$ and $R$ is the number of individuals of each group with ages between $x_{1}$ and $x_{2}$.

The dynamics of the disease propagation in the population are then described by the following set of hyperbolic PIDEs on $\Pi:=[0,+\infty) \times[0, L]$ (see [2, Chapter 1] and also [17, Chapter III] for detailed presentation of such systems)

$$
\begin{aligned}
& \partial_{t} S(t, x)+\partial_{x} S(t, x)+\mu(x) S(t, x)+\mathcal{G}[S(t), I(t)](x)=0, \\
& \partial_{t} I(t, x)+\partial_{x} I(t, x)+(\gamma(x)+\mu(x)) I(t, x) \\
& -\mathcal{G}[S(t), I(t)](x)=0, \\
& \partial_{t} R(t, x)+\partial_{x} R(t, x)+\mu(x) R(t, x)-\gamma(x) I(t, x)=0,
\end{aligned}
$$

where $\mathcal{G}[S(t), I(t)](x):=\beta(x) S(t, x) \int_{0}^{L} I(t, s) \mathrm{d} s$ represents the disease transmission rate by contact between susceptible and infected individuals which is assumed to be proportional to the sizes of both groups. $\beta(x)>0$ is the age-dependent transmission coefficient between all infected individuals and susceptibles having age $x$. The maximal life duration in the considered population is denoted by $L$ and, thus, $S(t, L)=I(t, L)=R(t, L)=0$. Parameter $\mu(x)>0$ denotes the natural age-dependent per capita death rate in the population and $\gamma(x)>0$ is the age-dependent rate at which infected individuals recover from the disease. Parameters $\mu, \beta$, and $\gamma$ are assumed twice continuously differentiable.

We consider the following boundary conditions

$$
S(t, 0)=B(t), \quad I(t, 0)=0, \quad R(t, 0)=0 .
$$

Here, $B(t)$ stands for the inflow of newborn individuals in the susceptible part of the population at time $t$. Let us assume that the number of people in the group $R$ of recovered patients 
between ages 0 and $x$, for every age $x \in[0, L]$ and time $t \geq 0$, is the system's output

$$
y(t, x)=\int_{0}^{x} R(t, s) \mathrm{d} s
$$

This is consistent with the remarks in the recent paper [29] about the COVID-19 pandemic. An alternative measurement, however, can be the number of infected patients $\int_{0}^{x} I(t, s) \mathrm{d} s$.

We perform the following coordinates invertible transformation, in order to write (49) in the appropriate form for observer design

$$
\begin{aligned}
& \xi_{1}(t, x)=\int_{0}^{x} R(t, s) \mathrm{d} s, \\
& \xi_{2}(t, x)=\int_{0}^{x} \gamma(s) I(t, s) \mathrm{d} s, \\
& \xi_{3}(t, x)=\int_{0}^{x} \beta(s) \gamma(s) S(t, s) \mathrm{d} s \int_{0}^{L} I(t, s) \mathrm{d} s .
\end{aligned}
$$

Using these coordinates, (49) is written as follows:

$$
\begin{aligned}
& \partial_{t} \xi_{1}(t, x)+\partial_{x} \xi_{1}(t, x)=\xi_{2}(t, x) \\
& +\int_{0}^{x} \mu^{\prime}(s) \xi_{1}(t, s) \mathrm{d} s-\mu(x) \xi_{1}(t, x), \\
& \partial_{t} \xi_{2}(t, x)+\partial_{x} \xi_{2}(t, x)=\xi_{3}(t, x) \\
& +\int_{0}^{x}\left(\kappa_{1}^{\prime}(s)-\kappa_{2}^{\prime}(s)\right) \xi_{2}(t, s) \mathrm{d} s+\left(\kappa_{2}(x)-\kappa_{1}(x)\right) \xi_{2}(t, x), \\
& \partial_{t} \xi_{3}(t, x)+\partial_{x} \xi_{3}(t, x)=\xi_{3}(t, x) g_{1}\left[\xi_{2}(t), \xi_{3}(t)\right](x) \\
& -\int_{0}^{x}\left(k_{3}^{\prime}(s)-\mu^{\prime}(s)-g_{2}\left[\xi_{2}(t)\right](L) \beta^{\prime}(s)\right) \xi_{3}(t, s) \mathrm{d} s \\
& +g_{2}\left[\xi_{2}(t)\right](L) B(t) \beta(0) \gamma(0), \\
& y(t, x)=C \xi(t, x), \\
& \xi(t, 0)=0,
\end{aligned}
$$

where

$\kappa_{1}(x):=\mu(x)+\gamma(x), \kappa_{2}(x):=\frac{\gamma^{\prime}(x)}{\gamma(x)}, \kappa_{3}(x):=\frac{(\beta(x) \gamma(x))^{\prime}}{\beta(x) \gamma(x)}$, $g_{1}\left[\xi_{2}(t), \xi_{3}(t)\right](x):=\kappa_{3}(x)-\mu(x)-\beta(x) g_{2}\left[\xi_{2}(t)\right](L)$

$$
\begin{aligned}
& +\frac{1}{g_{2}\left[\xi_{2}(t)\right](L)}\left(\int_{0}^{L}\left(\frac{\kappa_{1}(s)}{\gamma(s)}\right)^{\prime} \xi_{2}(t, s) \mathrm{d} s\right. \\
& \left.-\frac{\kappa_{1}(L)}{\gamma(L)} \xi_{2}(t, L)+\int_{0}^{L} \frac{\gamma^{\prime}(s)}{\gamma^{2}(s)} \xi_{3}(t, s) \mathrm{d} s+\frac{\xi_{3}(t, L)}{\gamma(L)}\right), \\
& g_{2}\left[\xi_{2}(t)\right](L):=\frac{\xi_{2}(t, L)}{\gamma(L)}+\int_{0}^{L} \frac{\gamma^{\prime}(s)}{\gamma^{2}(s)} \xi_{2}(t, s) \mathrm{d} s, \\
& C=\left(\begin{array}{lll}
1 & 0 & 0
\end{array}\right) .
\end{aligned}
$$

Notice that hyperbolic dynamics (53) of the transformed system satisfy a simpler version of structure (1a) (semilinear, with $\lambda=1)$, with $\mathcal{H}=0$ in (2b). Note also, that $g_{2}\left[\xi_{2}(t)\right](L)$, being equal to $\int_{0}^{L} I(t, b) \mathrm{d} b$, is positive, because of the positiveness of system (53) and, therefore, mapping $g_{1}$ is well defined.

Choose now some initial conditions, such that Assumption 1 of previous section is satisfied for system (53) with $\delta=$ 20 , we meet sufficient conditions for solvability of the $\mathrm{H}$ GODP for this particular choice of initial conditions (existence

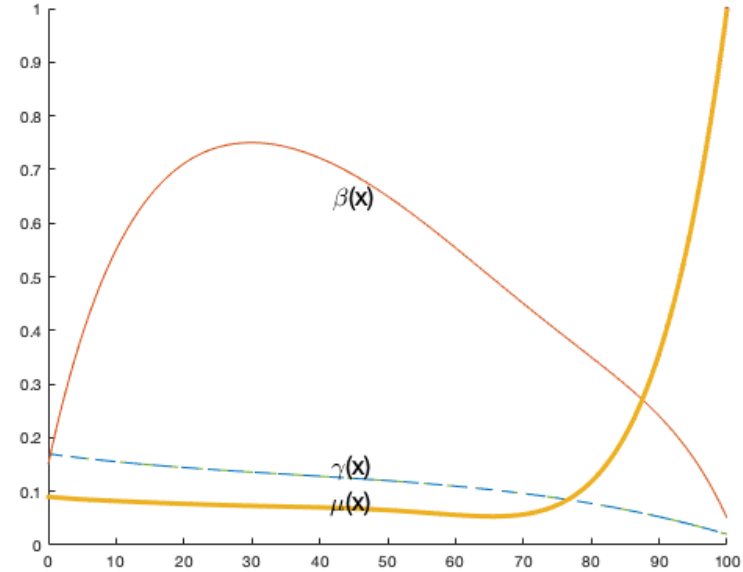

Fig. 1. Transmission, recovery, and death rates as functions of age

and uniqueness of classical solutions for (49) is established in [18]).

The high-gain observer dynamics, as in (7), are given by

$$
\begin{aligned}
& \partial_{t} \hat{\xi}_{1}(t, x)+\partial_{x} \hat{\xi}_{1}(t, x)=\hat{\xi}_{2}(t, x)+\int_{0}^{x} \mu^{\prime}(s) \hat{\xi}_{1}(t, s) \mathrm{d} s \\
& -\mu(x) \hat{\xi}_{1}(t, x)-\theta k_{1}\left(y(t, x)-\hat{\xi}_{1}(t, x)\right) \\
& \partial_{t} \hat{\xi}_{2}(t, x)+\partial_{x} \hat{\xi}_{2}(t, x)=\hat{\xi}_{3}(t, x) \\
& +\int_{0}^{x}\left(\kappa_{1}^{\prime}(s)-\kappa_{2}^{\prime}(s)\right) \hat{\xi}_{2}(t, s) \mathrm{d} s+\left(\kappa_{2}(x)-\kappa_{1}(x)\right) \hat{\xi}_{2}(t, x) \\
& -\theta^{2} k_{2}\left(y(t, x)-\hat{\xi}_{1}(t, x)\right) \\
& \partial_{t} \hat{\xi}_{3}(t, x)+\partial_{x} \hat{\xi}_{3}(t, x)=s_{\delta}^{3}\left(\hat{\xi}_{3}(t, x)\right) g_{1}\left[s_{\delta}^{2}\left(\hat{\xi}_{2}(t)\right), s_{\delta}^{3}\left(\hat{\xi}_{3}(t)\right)\right](x) \\
& -\int_{0}^{x}\left(k_{3}^{\prime}(s)-\mu^{\prime}(s)-g_{2}\left[s_{\delta}^{2}\left(\hat{\xi}_{2}(t)\right)\right](L) \beta^{\prime}(s)\right) s_{\delta}^{3}\left(\hat{\xi}_{3}(t, s)\right) \mathrm{d} s \\
& +g_{2}\left[\hat{\xi}_{2}(t)\right](L) B(t) \beta(0) \gamma(0)-\theta^{3} k_{3}\left(y(t, x)-\hat{\xi}_{1}(t, x)\right) \\
& \hat{\xi}(t, 0)=0
\end{aligned}
$$

where $s_{\delta}^{i}\left(\hat{\xi}_{i}\right), i=2,3$ are given by (4). We assume for the sake of simplicity that the inflow of the newborns $B(t)$ is not time-varying, but zero. This time invariance simplification is made in order to meet system's (1a) time invariance. The case where $B$ is time-varying is not included in this framework, but it is not hard to adjust the present analysis to this case.

In Figure 1 we represent the considered functions $\mu, \gamma$, and $\beta$ (academic choices) and we choose $L=100$.

We now apply Theorem 1 , with $\theta=20$ and $K=$ $\left(\begin{array}{lll}k_{1} & k_{2} & k_{3}\end{array}\right)^{\top}=\left(\begin{array}{lll}-2 & -1 & -1\end{array}\right)^{\top}$, which establishes the solvability of the H-GODP for system (53) with observer (54). After inversing the coordinates transformation, the convergence of the observation errors between states $S, I, R$ and $\hat{S}, \hat{I}, \hat{R}$ (the observer states in the original coordinates) is guaranteed by Theorem 1 .

In Figure 2 we represent the output $\xi_{1}$. In Figures 3 - 5 we see the observation errors for each of the original states $S, I$ and $R$, after choosing arbitrary observer's initial conditions satisfying compatibility conditions.

Remark 8: In Theorem 1 we proved a type of stability in the $C^{1}$ spatial norm. It turns out that this is essential for the 


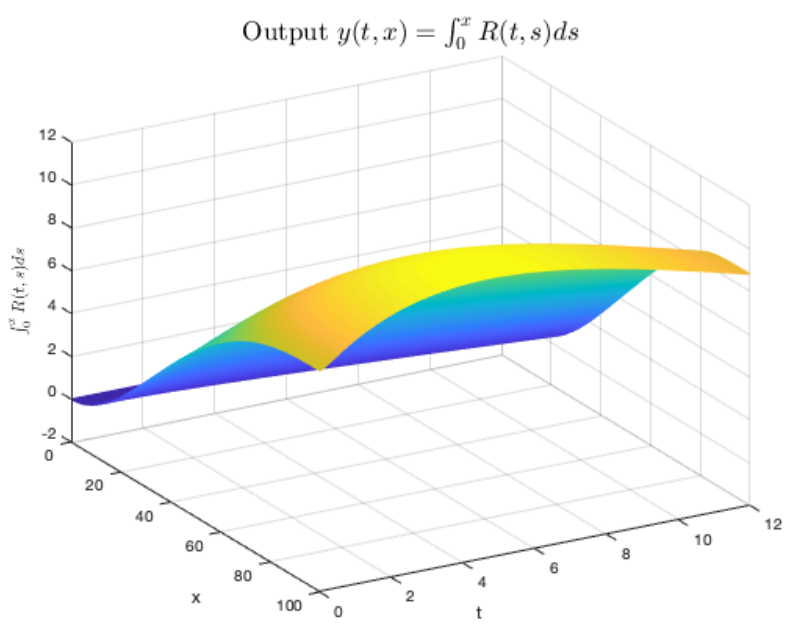

Fig. 2. Time and age evolution of simulated system's output

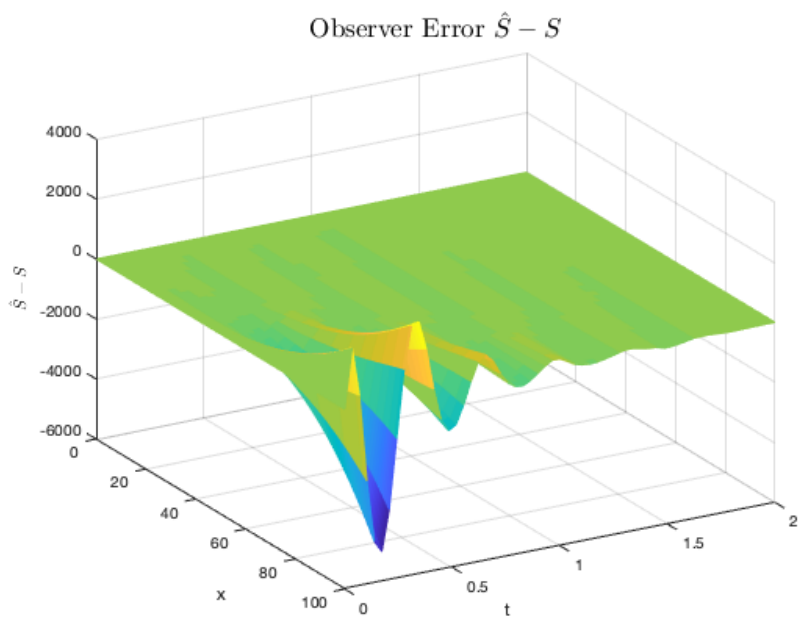

Fig. 3. Time and age evolution of the first estimation error

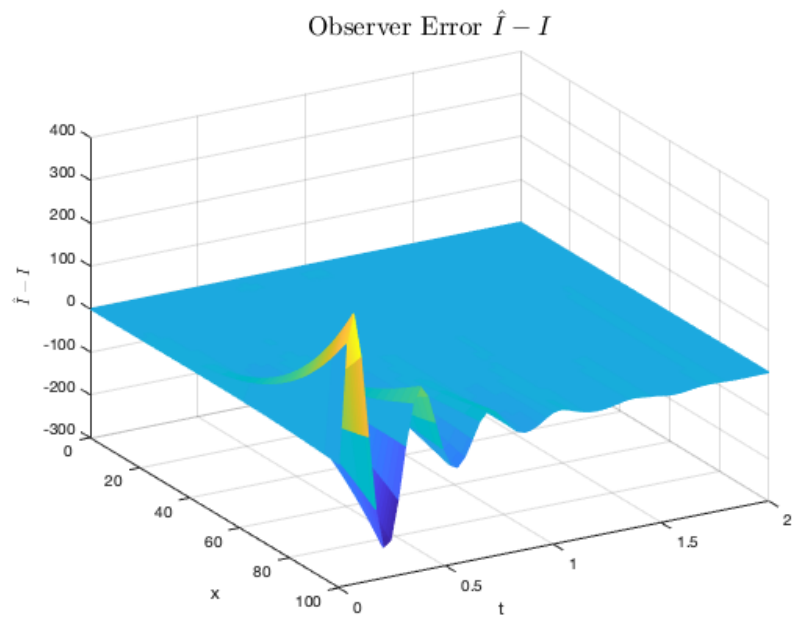

Fig. 4. Time and age evolution of the second estimation error

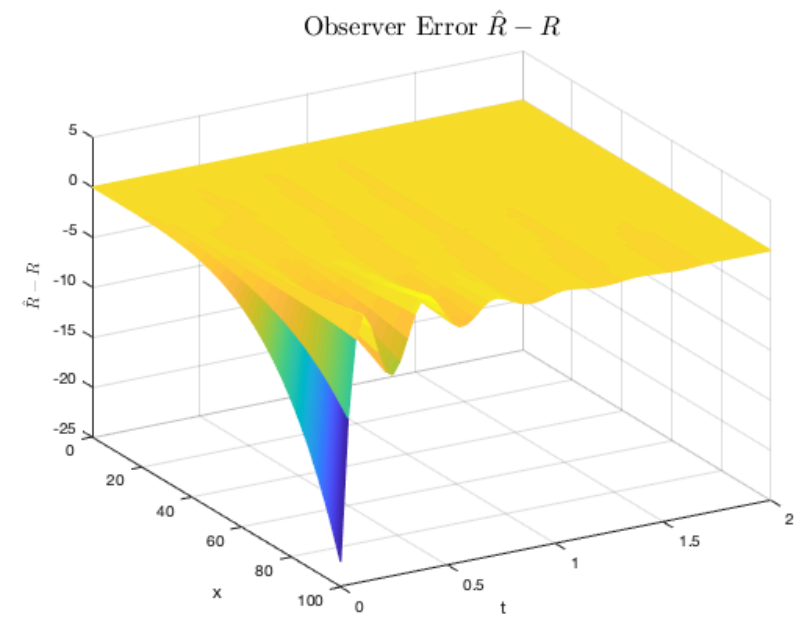

Fig. 5. Time and age evolution of the third estimation error

particular case of systems that we study in the current section and it is due to the nonlocal transformation we performed in (52). More precisely, the exponential stability of the spatial derivative of the error between (53) and (54) induces the exponential stability in the sup-norm for the estimation error of the initial system (49).

\section{ACKNOWLEDGEMENT}

The first author would like to thank Fabian Wirth (University of Passau) and Roxanne Jackson for fruitful discussions on this topic.

\section{CONCLUSION}

In this paper we designed a high-gain observer for a class of observable systems of hyperbolic PIDEs with distributed measurement. This result constitutes an extension of the highgain observer design for finite-dimensional systems to a class of PIDEs and, also, an extension of our previous works towards this direction. We proved the exponential decay of the observer error in the $C^{1}$ - norm step by step by first choosing an appropriate Lyapunov functional and we illustrated our methodology with its application to an epidemic model. The extension of this methodology to more general cases of hyperbolic systems with distinct characteristic velocities will be subject to our future work.

\section{REFERENCES}

[1] F. Alabau-Boussouira, J.-M. Coron, and G. Olive, "Internal controllability of first order quasilinear hyperbolic systems with a reduced number of controls," SIAM J. Control and Optimization, vol. 55, no. 1, pp. 300323, 2017.

[2] G. Bastin and J.-M. Coron, "Stability and boundary stabilization of 1-d hyperbolic systems," Progress in Nonlinear Differential Equations and Their Applications. Springer International Publishing, 2016.

[3] G. Besançon, D. Georges, and Z. Benayache, "Sur la commande en dimension finie d'une classe de systèmes non linéaires de dimension infinie," Conf. Internationale Francophone d'Automatique, Bordeaux, France, 2006.

[4] G. Besançon, B. Pham, and D. Georges, "Robust state estimation for a class of convection-diffusion-reaction systems," IFAC Workshop on Control of Systems Governed by partial Differential Equations, Paris, France, 2013. 
[5] D. Boskovic and M. Krstic, "Backstepping control of chemical tubular reactors," Computers and Chemical Engineering, vol. 26, pp. 10771085, 2002.

[6] F. Castillo, E. Witrant, C. Prieur, and L. Dugard, "Boundary observers for linear and quasi-linear hyperbolic systems with application to flow control," Automatica, vol. 49, no. 11, pp. 3180-3188, 2013.

[7] P. D. Christofides and P. Daoutidis, "Feedback control of hyperbolic pde systems," AIChE Journal, vol. 42, no. 11, pp. 3063-3086, 1996.

[8] J.-M. Coron and G. Bastin, "Dissipative boundary conditions for onedimensional quasi-linear hyperbolic systems: Lyapunov stability for the C1-norm," SIAM J. Control Optim., vol. 53, no. 3, pp. 1464-1483, 2015.

[9] J.-M. Coron, L. Hu, and G. Olive. "Stabilization and controllability of first-order integro-differential hyperbolic equations," Journal of Functional Analysis, Elsevier, vol. 271, no. 12, pp. 3554-3587, 2016.

[10] J.-M. Coron, R. Vazquez, M. Krstic, and G. Bastin, "Local exponential stabilization of a $2 \times 2$ quasilinear hyperbolic system using backstepping," SIAM J. Control Optim., vol. 51, no. 3, pp. 2005-2035, 2013.

[11] R. Curtain, "Finite-dimensional compensator design for parabolic distributed systems with point sensors and boundary input," IEEE Transactions on Automatic Control, vol. 27, no. 1, pp. 98-104, 1982.

[12] A. Deutschmann, L. Jadachowski, and A. Kugi, "Backstepping-based boundary observer for a class of time-varying linear hyperbolic PIDEs," Automatica, pp. 369-377, 2016.

[13] F. Di Meglio, R. Vazquez, and M. Krstic, "Stabilization of a system of $n+1$ coupled first-order hyperbolic linear PDEs with a single boundary input," IEEE Transactions on Automatic Control, vol. 58, pp. 30973111, 2013.

[14] J. P. Gauthier and G. Bornard, "Observability for any $u(t)$ of a class of nonlinear systems," IEEE Transactions on Automatic Control, vol. 26, no. 4, pp. 922-926, 1981

[15] J. P. Gauthier, H. Hammouri, and S. Othman, "A simple observer for nonlinear systems: applications to bioreactors," IEEE Transactions on Automatic Control, vol. 37, no. 6, pp. 875-880, 1992.

[16] A. Hasan, O. M. Aamo, and M. Krstic, "Boundary observer design for hyperbolic PDE-ODE cascade systems," Automatica, vol. 68, pp.75-86, 2016.

[17] M. Iannelli, "Mathematical Theory of Age-Structured Population Dynamics," Appl. Math. Monogr. C.N.R., 1995.

[18] H Inaba, "Mathematical analysis of an age-structured SIR epidemic model with vertical transmission," Discrete Contin. Dyn. Syst. Ser. B, vol. 6, pp.69-96, 2006.

[19] I. Karafyllis and M. Krstic, "Stability of integral delay equations and stabilization of age-structured models," ESAIM: Control, Optimisation and Calculus of Variations, vol. 23, pp.1667-1714, 2017.

[20] W. O. Kermack and A. G. McKendrick, "A contribution to the mathematical theory of epidemics," Proceedings of the Royal Society of London Series A, vol. 115, pp. 700-721, 1927.

[21] H. K. Khalil, "High-gain observers in nonlinear feedback control," Advances in Design and Control. SIAM, 2017.

[22] C. Kitsos, G. Besançon, and C. Prieur "High-gain observer design for a class of hyperbolic systems of balance laws," IEEE Conference on Decision and Control, Miami, USA, 2018.

[23] C. Kitsos, G. Besançon, and C. Prieur "A high-gain observer for a class of $2 \times 2$ hyperbolic systems with $\mathrm{C} 1$ exponential convergence," IFAC Workshop on Control of Systems Governed by Partial Differential Equations, Oaxaca, Mexico, 2019.

[24] C. Kitsos, G. Besançon, and C. Prieur "High-gain observers for a class of $2 \times 2$ quasilinear hyperbolic systems with 2 different velocities," IFAC Workshop on Nonlinear Control Systems, Vienna, Austria, 2019.

[25] I. Kmit, "Classical solvability of nonlinear initial-boundary problems for first-order hyperbolic systems," International Journal of Dynamical Systems and Differential Equations, vol. 1, no. 3, pp. 191-195, 2008.

[26] T-T. Li, "Boundary value problems for quasilinear hyperbolic systems," Duke University Mathematical series, V. Duke University Mathematics Department, Durham, NC, 1985.

[27] T-T. Li, "Exact boundary observability for quasilinear hyperbolic systems," ESAIM: Control, Optimisation and Calculus of Variations, vol. 14, no. 4, pp. 759-766, 2008.

[28] V. Nguyen, D. Georges, and G. Besançon, "State and parameter estimation in 1-d hyperbolic PDEs based on an adjoint method," Automatica, vol. 67, pp. 185-191, 2016

[29] S. W. Park and B. M. Bolker, "A Note on Observation Processes in Epidemic Models," Bull. Math. Biol., vol. 82 (3), 2020

[30] C. Prieur, A. Girard, and E. Witrant, "Stability of switched linear hyperbolic systems by Lyapunov techniques," IEEE Transactions on Automatic Control, vol. 59, no. 8, pp. 2196-2202, 2014.
[31] A. Schaum, J. A. Moreno, J. Alvarez, and T. Meurer, "A simple observer scheme for a class of 1-D semi-linear parabolic distributed parameter systems," European Control Conf., Linz, Austria, pp. 49-54, 2015.

[32] K. Schmidt, I. Karafyllis, and M. Krstic, "Yield trajectory tracking for hyperbolic age-structured population systems," Automatica, vol. 90, pp. 138-146, 2018.

\section{APPENDIX A PROOF OF LEMMA 1}

To prove the existence and uniquenes of global classical solutions to the observer system, which is a semilinar system of hyperbolic PIDEs, we follow a fixed-point methodology inspired by [25] (Theorem 2.1), where a similar result is proven for a system of semilinear hyperbolic PDEs.

We first represent (7) in its integral form, as follows:

$$
\begin{aligned}
\hat{\xi}(t, x)= & \mathcal{G}[\hat{\xi}](t, x) ; \\
\mathcal{G}[\hat{\xi}](t, x):= & \Phi\left(t, t_{0}(t, x)\right) \mathcal{R}[\hat{\xi}](t, x)+\int_{t_{0}(t, x)}^{t} \Phi(t, s) \\
& \times\left(f\left(\omega(s ; t, x), \mathcal{F}\left[s_{\delta}(\hat{\xi}(s))\right](\omega(s ; t, x))\right)\right. \\
& -\Theta K y(s, \omega(s ; t, x))) \mathrm{d} s \\
\mathcal{R}[\hat{\xi}](t, x)= & \begin{cases}\hat{\xi}^{0}(\omega(0 ; t, x)), & \text { when } t_{0}(t, x)=0 \\
\mathcal{H}\left(s_{\delta}\left(\hat{\xi}\left(t_{0}(t, x), L\right)\right)\right), & \text { when } t_{0}(t, x) \neq 0\end{cases}
\end{aligned}
$$

where $\omega(s ; t, x)$ denotes the characteristic (for characteristic velocity $\lambda \circ y$ ) passing through $(t, x) \in \Pi, t_{0}(t, x)$ is the smallest value of $s$ at which $x_{0}=\omega(s ; t, x)$ reaches $\partial \Pi$ and by $\Phi\left(t, t_{0}\right)$ we denote the fundamental matrix of $A+\Theta K C$. More precisely, $\omega(s ; t, x)$ is the unique solution of

$$
\left\{\begin{array}{l}
\partial_{s} \omega(s ; t, x)=(\lambda \circ y)(s, \omega(s ; t, x)) \\
\omega(t ; t, x)=x
\end{array}\right.
$$

It is sufficient to prove this lemma in $\Pi^{T}:=(0, T) \times(0, L)$ for some fixed $T>0$.

In conjunction with our regularity assumptions, it is easy to see that for every $\delta>0$, there exist $L_{f}, L_{\mathcal{H}} \geq 0$, such that

$$
\begin{array}{r}
\sup _{z \in C^{0}\left(\Pi ; \mathbb{R}^{n}\right)}\left\{\left\|\left.D_{u} f(\cdot, \mathcal{F}[u])\right|_{u=s_{\delta}(z)}\right\| \|_{\text {op }}\left|D_{z} s_{\delta}(z)\right|\right\} \leq L_{f}, \\
\sup _{z \in C^{0}\left(\Pi ; \mathbb{R}^{n}\right)}\left|D_{u} \mathcal{H}(u)\right|_{u=s_{\delta}(z)} D_{z} s_{\delta}(z) \mid \leq L_{\mathcal{H}},
\end{array}
$$

where $\|\cdot\|_{\text {op }}:=\|\cdot\|_{\mathcal{L}\left(C^{0}\left(\Pi ; \mathbb{R}^{n}\right) ; C^{0}\left(\Pi ; \mathbb{R}^{n}\right)\right)} \cdot$

First, we prove that there exists a unique continuous solution in $\Pi^{\tau_{0}}$, for some $\tau_{0}>0$, such that:

$$
\omega(t ; \tau, 0)<L ; \tau>0, t \in\left[\tau, \tau+\tau_{0}\right] .
$$

From the integral representation (55) we obtain the following equation for $t$ in $\left[0, \tau_{0}\right]$ :

$$
\begin{aligned}
\hat{\xi}(t, L)= & \Phi(t, 0) \hat{\xi}^{0}(\omega(0 ; t, L))+\int_{0}^{t} \Phi(t, s) \\
& \times\left(f\left(\omega(s ; t, L), \mathcal{F}\left[s_{\delta}(\hat{\xi}(s))\right](\omega(s ; t, L))\right)\right. \\
& -\Theta K y(s, \omega(s ; t, L))) \mathrm{d} s .
\end{aligned}
$$


Now, choosing $\hat{\xi}^{1}, \hat{\xi}^{2}$ in $C^{0}\left(\overline{\Pi^{\tau_{0}}} ; \mathbb{R}^{n}\right)$ with identical initial conditions, from the mean value theorem, we obtain the following:

$$
\begin{aligned}
& \sup _{(t, x) \in \overline{\Pi^{\tau_{0}}}} \mid f\left(x, \mathcal{F}\left[s_{\delta}\left(\hat{\xi}^{1}(t)\right)\right](x)\right) \\
& -f\left(x, \mathcal{F}\left[s_{\delta}\left(\hat{\xi}^{2}(t)\right)\right](x)\right) \mid \\
& \leq \sup _{s \in[0,1]}\left\{\| D_{u} f\left(\cdot,\left.\mathcal{F}\left[s_{\delta}(u)\right]\right|_{\left.u=\hat{\xi}^{1}+s\left(\hat{\xi}^{2}-\hat{\xi}^{1}\right)\right) \|_{\mathrm{op}}}\right.\right. \\
& \left.\times\left|D_{u} s_{\delta}(u)\right|_{u=\hat{\xi}^{1}+s\left(\hat{\xi}^{2}-\hat{\xi}^{1}\right)} \mid\right\} \sup _{(t, x) \in \Pi^{\tau_{0}}}\left|\hat{\xi}^{1}(t, x)-\hat{\xi}^{2}(t, x)\right| \\
& \leq L_{f} \sup _{(t, x) \in \bar{\Pi}^{\tau_{0}}}\left|\hat{\xi}^{1}(t, x)-\hat{\xi}^{2}(t, x)\right| .
\end{aligned}
$$

This, in conjunction with (55), directly leads to the following:

$$
\begin{aligned}
& \sup _{(t, x) \in \overline{\Pi^{\tau} \tau_{0}}}\left|\mathcal{G}\left[\hat{\xi}^{1}\right]-\mathcal{G}\left[\hat{\xi}^{2}\right]\right| \leq \tau_{0} q_{0} \sup _{(t, x) \in \overline{\Pi^{\tau_{0}}}}\left|\hat{\xi}^{1}-\hat{\xi}^{2}\right| ; \\
& q_{0}:=\max _{(t, x) \in \overline{\Pi^{\tau_{0}}}}\left|\Phi\left(t, t_{0}(t, x)\right)\right| L_{f}\left(1+L_{\mathcal{H}}\right) .
\end{aligned}
$$

Now, choosing $\tau_{0}=\left(2 q_{0}\right)^{-1}$ and applying the Banach fixedpoint theorem, we prove the existence and uniqueness of a $C^{0}\left(\overline{\Pi^{\tau_{0}}} ; \mathbb{R}^{n}\right)$ solution $\hat{\xi}$. In order to prove the existence and uniqueness of a continuous solution in $\Pi^{T}$, for arbitrary $T$, we follow the previous procedure in $\left\lceil T / \tau_{0}\right\rceil$ steps, iterating the local existence-uniqueness result in domains $\left(\Pi^{j \tau_{0}} \cap \Pi^{T}\right) \backslash$ $\overline{\Pi^{(j-1) \tau_{0}}}$, where $j \leq\left\lceil T / \tau_{0}\right\rceil$.

Next, we follow the same procedure to prove the existenceuniqueness of $C^{1}$ solutions in $\Pi^{T}$, by considering the problem for $\hat{\xi}_{x}$.

By (55), we obtain:

$$
\begin{gathered}
\hat{\xi}_{x}(t, x)=\frac{\mathrm{d}}{\mathrm{d} x} \Phi\left(t, t_{0}(t, x)\right) \mathcal{R}[\hat{\xi}](t, x) \\
+\Phi\left(t, t_{0}(t, x)\right) \mathcal{R}_{1}[\hat{\xi}](t, x)+\int_{t_{0}(t, x)}^{t} \Phi(t, s) \\
\times\left[\left(\left\langle\left.D_{u} f(z, \mathcal{F}[u])\right|_{u=s_{\delta}(\hat{\xi}(s))(z)}, D_{\hat{\xi}} s_{\delta}(\hat{\xi}(s))(z)\right.\right.\right. \\
\left.\times \hat{\xi}_{z}(s, z)\right\rangle-\partial_{z}(\lambda \circ y)(s, z) \hat{\xi}_{z}(s, z) \\
\left.\left.-\Theta K y_{z}(s, z)\right)\right]\left.\right|_{z=\omega(s ; t, x)} \mathrm{d} s, \\
\mathcal{R}_{1}[\hat{\xi}](t, x)=\left.\frac{\mathrm{d}}{\mathrm{d} u} \hat{\xi}^{0}(u)\right|_{u=\omega(0 ; t, x)}, \text { when } t_{0}(t, x)=0 \\
\times\left(f\left(0, \mathcal{F}\left[s_{\delta}(\hat{\xi}(\tau))\right](0)\right)-\left.D_{u} \mathcal{H}(u)\right|_{u=s_{\delta}(\hat{\xi})}(\tau, L)\right. \\
\left.\times D_{\hat{\xi}} s_{\delta}(\hat{\xi}(\tau))(L) \hat{\xi}_{\tau}(\tau, L)\right)\left.\right|_{\tau=t_{0}(t, x)}, \text { when } t_{0}(t, x) \neq 0 .
\end{gathered}
$$

We prove the existence-uniqueness of continuous solutions $\hat{\xi}_{x}$ in $\overline{\Pi^{\tau}}$, for some $\tau_{1}$ satisfying condition (57) with $\tau_{0}$ replaced by $\tau_{1}$. Combining observer system's dynamics (7a) and (59), we get the following expression for the right boundary:

$$
\begin{gathered}
\hat{\xi}_{t}(t, L)=-(\lambda \circ y)(t, L) \hat{\xi}_{x}(t, L)+A \hat{\xi}(t, L) \\
-\Theta K(y(t, L)-C \hat{\xi}(t, L))+f\left(L, \mathcal{F}\left[s_{\delta}(\hat{\xi}(t))\right](L)\right) \\
=-(\lambda \circ y)(t, L)\left(\left.\Phi(t, 0) \frac{\mathrm{d}}{\mathrm{d} u} \hat{\xi}^{0}(u)\right|_{u=\omega(0 ; t, L)}\right. \\
+\int_{0}^{t} \Phi(t, s)\left[\left(\left\langle\left.D_{u} f(z, \mathcal{F}[u])\right|_{u=s_{\delta}(\hat{\xi}(s))(z)},\right.\right.\right. \\
\left.D_{\hat{\xi}} s_{\delta}(\hat{\xi}(s))(z) \hat{\xi}_{z}(s, z)\right\rangle-\partial_{z}(\lambda \circ y)(s, z) \hat{\xi}_{z}(s, z) \\
\left.\left.\left.-\Theta K y_{z}(s, z)\right)\right]\left.\right|_{z=\omega(s ; t, L)} \mathrm{d} s\right)+(A+\Theta K C) \hat{\xi}(t, L) \\
-\Theta K y(t, L)+f\left(L, \mathcal{F}\left[s_{\delta}(\hat{\xi}(t))\right](L)\right) .
\end{gathered}
$$

Taking into account that $\hat{\xi}$ is a known continuous function, we apply the operator defined by the right hand side of (59) to continuous functions $\partial_{x} \hat{\xi}^{1}$ and $\partial_{x} \hat{\xi}^{2}$, starting from the same initial conditions. We can, therefore, obtain the estimate

$$
\begin{gathered}
\sup _{(t, x) \in \overline{\Pi^{\tau_{1}}}}\left|\partial_{x} \hat{\xi}^{1}-\partial_{x} \hat{\xi}^{2}\right| \leq \tau_{1} q_{1} \sup _{(t, x) \in \overline{\Pi^{\tau_{1}}}}\left|\partial_{x} \hat{\xi}^{1}-\partial_{x} \hat{\xi}^{2}\right| ; \\
q_{1}:=\max _{(t, x) \in \overline{\Pi^{\tau_{1}}}}\left|\Phi\left(t, t_{0}(t, x)\right)\right|\left(L_{f}\right. \\
\left.+\max _{(t, x) \in \overline{\Pi^{\tau_{1}}}} \partial_{x}|(\lambda \circ y)(t, x)|\right)\left(1+L_{\mathcal{H}} \max _{(t, x) \in \overline{\bar{\Pi}^{\tau_{1}}}}((\lambda \circ y)(t, x))\right. \\
\left.\times \max _{(t, x) \in \bar{\Pi}^{\tau_{1}}}((\lambda \circ y)(t, x))^{-1}\right) .
\end{gathered}
$$

Choosing $\tau_{1}$, such that $q_{1} \tau_{1}<1$ and in conjuction with the fact that $\hat{\xi}$ is $C^{1}$ function in both arguments (as a result of (7a)), we obtain the existence-uniqueness of classical solutions in $\overline{\Pi^{\tau_{1}}}$. Iterating the previous local existence-uniqueness result in domains $\left(\Pi^{j \tau_{1}} \cap \Pi^{T}\right) \backslash \overline{\Pi^{(j-1) \tau_{1}}}$, where $j \leq\left\lceil T / \tau_{1}\right\rceil$, we obtain classical solutions in $\Pi^{T}$ and since $T$ is arbitrary, we obtain unique classical solutions in $\Pi$. The proof is complete.

\section{APPENDIX B}

PROPERTIES OF THE LYAPUNOV FUNCTIONAL

We prove here two properties, which we have invoked in the stability proof of Theorem 1 .

Property 1: For every continuous functions (or more generally, $L^{\infty}$ functions, where $\|\cdot\|_{\infty}$ denotes the ess.sup-norm) $\varepsilon, \varepsilon_{t}:[0, L] \rightarrow \mathbb{R}^{n} ; L>0$, matrix $P, \pi:[0, L] \rightarrow \mathbb{R}$ as in (22), $\rho_{0}>0$, and $\mu$ in $\mathbb{R}$, we have

$$
\lim _{p \rightarrow+\infty} W_{p}=\left\|e^{\mu \cdot}\left(\varepsilon^{\top} P \varepsilon+\rho_{0} \varepsilon_{t}^{\top} P \varepsilon_{t}\right)\right\|_{\infty}
$$

where $W_{p}$, as in (21a), is given by

$$
\begin{aligned}
W_{p} & :=\left(\int_{0}^{L} \pi(x) e^{p \mu x} G_{p}(x) \mathrm{d} x\right)^{1 / p} ; \\
G_{p} & :=\left(\varepsilon^{\top} P \varepsilon+\rho_{0} \varepsilon_{t}^{\top} P \varepsilon_{t}\right)^{p} .
\end{aligned}
$$


To prove this, define, first for $\bar{\epsilon}>0$ arbitratily small, the set

$$
\begin{aligned}
& B_{\bar{\epsilon}}:=\left\{x \in[0, L]:\left|e^{\mu x}\left(\varepsilon^{\top}(x) P \varepsilon(x)+\rho_{0} \varepsilon_{t}^{\top}(x) P \varepsilon_{t}(x)\right)\right|\right. \\
& \geq\left\|e^{\mu \cdot}\left(\varepsilon^{\top} P \varepsilon+\rho_{0} \varepsilon_{t}^{\top} P \varepsilon_{t}\right)\right\|_{\infty}-\bar{\epsilon} ; \\
& \left.\bar{\epsilon}<\left\|e^{\mu \cdot}\left(\varepsilon_{t} P \varepsilon+\varepsilon_{t} P \varepsilon_{t}\right)\right\|_{\infty}\right\} .
\end{aligned}
$$

Then, for all $p$ in $[1,+\infty)$,

$$
\begin{aligned}
W_{p} & \geq\left(\int_{B_{\bar{\epsilon}}}\left(\left\|e^{\mu \cdot}\left(\varepsilon^{\top} P \varepsilon+\rho_{0} \varepsilon_{t}^{\top} P \varepsilon_{t}\right)\right\|_{\infty}-\bar{\epsilon}\right)^{p} \mathrm{~d} x\right)^{1 / p} \\
& =\left(\left\|e^{\mu \cdot}\left(\varepsilon^{\top} P \varepsilon+\rho_{0} \varepsilon_{t}^{\top} P \varepsilon_{t}\right)\right\|_{\infty}-\bar{\epsilon}\right) \mu\left(B_{\bar{\epsilon}}\right)^{1 / p}
\end{aligned}
$$

where $\mu\left(B_{\bar{\epsilon}}\right)$ denotes the Lebesgue measure of $B_{\bar{\epsilon}}$.

The above gives

$$
\liminf _{p \rightarrow+\infty} W_{p} \geq\left\|e^{\mu \cdot}\left(\varepsilon^{\top} P \varepsilon+\rho_{0} \varepsilon_{t}^{\top} P \varepsilon_{t}\right)\right\|_{\infty} .
$$

Furthermore,

$$
W_{p} \leq\left\|e^{\mu \cdot}\left(\varepsilon^{\top} P \varepsilon+\rho_{0} \varepsilon_{t}^{\top} P \varepsilon_{t}\right)\right\|_{\infty}(L \bar{\pi})^{1 / p} .
$$

This implies

$$
\limsup _{p \rightarrow+\infty} W_{p} \leq\left\|e^{\mu \cdot}\left(\varepsilon^{\top} P \varepsilon+\rho_{0} \varepsilon_{t}^{\top} P \varepsilon_{t}\right)\right\|_{\infty}
$$

since $L \bar{\pi}$ is finite.

By virtue of (65) and (67), we obtain (61).

\section{Property 2:}

$\dot{W}_{p} \stackrel{*}{\rightarrow} \frac{\mathrm{d}}{\mathrm{d} t}\left(\left\|e^{\mu \cdot} \varepsilon^{\top} P \varepsilon\right\|_{\infty}+\left\|\rho_{0} e^{\mu \cdot} \varepsilon_{t}^{\top} P \varepsilon_{t}\right\|_{\infty}\right)$

in the weak ${ }^{*}$ topology $\sigma\left(L^{\infty}(0,+\infty), L^{1}(0,+\infty)\right)$,

as $p \rightarrow+\infty$

where by $\sigma\left(X, X^{*}\right)$ we denote the weak* topology on $X$, with $X^{*}$ being the topological dual of $X$.

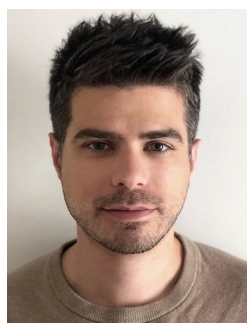

Constantinos Kitsos received a Diploma in Electrical and Computer Engineering and an M.Sc. in Applied Mathematics both from the National Technical University of Athens, Greece, in 2014 and 2016, respectively. In 2020, he received a Ph.D. degree at the Control Systems Department of University Grenoble Alples, Gipsa-lab, France. He is currently a postdoctoral researcher CNRS at the laboratory LAAS in Toulouse, France. His research interests include nonlinear observer design, control and inverse problems for partial differential equations.

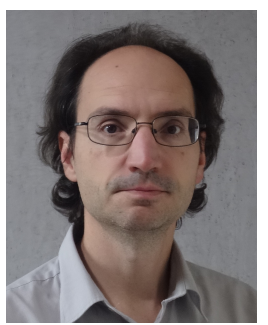

Gildas Besançon was born in 1971 in Toulouse, France. He received an Electrical Engineering diploma in 1993, and a PHD degree in Control in 1996, both in Grenoble, France. After a stay at Rome University La Sapienza, Italy, he joined the Institut National Polytechnique de Grenoble in 1998. He got the French degree of 'Habilitation à Diriger des Recherches' in 2002, and became a full professor at Grenoble INP in 2010, to which he is still currently affiliated. In 2010, he was also distinguished by becoming a member of the Institut Universitaire de France. His research activities are held at the Control Systems Department of Gipsa-lab in the area of nonlinear and complex systems, with a special interest in observer issues, and applications in energy, hydraulics, or micro/nanosciences.

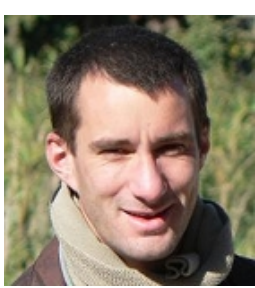

Christophe Prieur was born in Essey-les-Nancy, France, in 1974. He graduated in Mathematics from the Ecole Normale Supérieure de Cachan, France in 2000. He received the Ph.D. degree in 2001 in Applied Mathematics from the Université Paris-Sud, France. From 2002 he was an associate researcher CNRS at the laboratory SATIE, Cachan, France, and at the LAAS, Toulouse, France (2004-2010). In 2010 he joined the Gipsa-lab, Grenoble, France where he is currently a senior researcher of the CNRS. His current research interests include nonlinear control theory, hybrid systems, and control of partial differential equations. 